This is a postprint version of the following published document:

Lv, J.P., et al. The three-state Potts antiferromagnet on plane quadrangulations. In: Journal of Physics A: Mathematical and Theoretical, 51(36), 3650012, July 2018, 44 pp.

DOI: https://doi.org/10.1088/1751-8121/aad1fe

(C) 2018 IOP Publishing Ltd 


\title{
The three-state Potts antiferromagnet on plane quadrangulations
}

\author{
Jian-Ping Lv ${ }^{1,2}$, Youjin Deng ${ }^{3,4}$, Jesper Lykke Jacobsen ${ }^{5,6,7}$, and Jesús Salas ${ }^{8,9}$ \\ ${ }^{1}$ Department of Physics, Anhui Normal University, Wuhu 241000, China \\ ${ }^{2}$ Anhui Province Key Laboratory of Optoelectric Materials Science and Technology, \\ Wuhu 241000, China \\ phys.lv@gmail.com \\ ${ }^{3}$ Hefei National Laboratory for Physical Sciences at Microscale and \\ Department of Modern Physics, University of Science and Technology of China, \\ Hefei, Anhui 230026, China \\ ${ }^{4}$ CAS Center for Excellence and Synergetic Innovation Center in Quantum Information \\ and Quantum Physics, University of Science and Technology of China, \\ Hefei, Anhui 230026, China \\ yjdeng@ustc.edu.cn \\ ${ }^{5}$ Laboratoire de Physique Théorique, Département de Physique de l'ENS, \\ École Normale Supérieure, Sorbonne Université, CNRS, PSL Research University, \\ 75005 Paris, France \\ ${ }^{6}$ Sorbonne Université, École Normale Supérieure, CNRS, \\ Laboratoire de Physique Théorique (LPT ENS), 75005 Paris, France \\ ${ }^{7}$ Institut de Physique Théorique, CEA Saclay, 91191 Gif-sur-Yvette, France \\ jesper.jacobsen@ens.fr \\ ${ }^{8}$ Grupo de Modelización, Simulación Numérica y Matemática Industrial, \\ Universidad Carlos III de Madrid, Avda. de la Universidad 30, 28911 Leganés, Spain \\ ${ }^{9}$ Grupo de Teorías de Campos y Física Estadística, \\ Instituto Gregorio Millán, Universidad Carlos III de Madrid, \\ Unidad Asociada al Instituto de Estructura de la Materia, CSIC, Madrid, Spain \\ jsalas@math.uc3m.es
}

April 24, 2018

Revised July 03, 2018

\begin{abstract}
We study the antiferromagnetic 3-state Potts model on general (periodic) plane quadrangulations $\Gamma$. Any quadrangulation can be built from a dual pair $\left(G, G^{*}\right)$. Based on the duality properties of $G$, we propose a new criterion to predict the phase diagram of this model. If $\Gamma$ is of self-dual type (i.e., if $G$ is isomorphic to its dual $G^{*}$ ), the model has a zero-temperature critical point
\end{abstract}


with central charge $c=1$, and it is disordered at all positive temperatures. If $\Gamma$ is of non-self-dual type (i.e., if $G$ is not isomorphic to $G^{*}$ ), three ordered phases coexist at low temperature, and the model is disordered at high temperature. In addition, there is a finite-temperature critical point (separating these two phases) which belongs to the universality class of the ferromagnetic 3-state Potts model with central charge $c=4 / 5$. We have checked these conjectures by studying four (resp. seven) quadrangulations of self-dual (resp. non-self-dual) type, and using three complementary high-precision techniques: Monte-Carlo simulations, transfer matrices, and critical polynomials. In all cases, we find agreement with the conjecture. We have also found that the Wang-SwendsenKotecký Monte Carlo algorithm does not have (resp. does have) critical slowing down at the corresponding critical point on quadrangulations of self-dual (resp. non-self-dual) type.

Keywords: Duality, Potts antiferromagnet, plane quadrangulation, transfer matrix, Monte Carlo simulation, critical polynomial, Wang-Swendsen-Koteký algorithm. 


\section{Introduction}

The $q$-state Potts model $[62,82,83]$ plays an important role in the theory of critical phenomena, especially in two dimensions $[5,15,57]$, and has applications to various condensed-matter systems $[82,83]$. Ferromagnetic Potts models are by now fairly well understood, thanks to universality; and much is known about their phase diagrams $[82,83]$, critical exponents, and their relation to conformal field theories (CFT) $[15,57]$.

On the other hand, the behaviour of antiferromagnetic (AF) Potts models depends strongly on the microscopic lattice structure. One striking example is the AF 3-state Potts model:

- On the honeycomb lattice, it is disordered at all temperatures $T \geq 0$ [64].

- On the square lattice, it has a critical point at $T=0$ (i.e., at $T=0$, the model has algebraic decay of correlations), and it is disordered at any positive temperature $T>0[10,39,58,67]$.

- On the diced lattice, it displays an ordinary finite-temperature critical point [45].

- On the triangular lattice, it shows a finite-temperature weak first-order phase transition [1].

Therefore, many basic questions about the phase diagram of this AF model must be investigated on a case-by-case basis. The absence of universality is probably the main cause why our understanding of Potts antiferromagnets is less advanced than that of their ferromagnetic (FM) counterparts. However, for the last decades, many new numerical and theoretical works have endeavoured to understand the AF regime. As a matter of fact, some sort of universality has been recovered in this regime.

In particular, one expects that for each lattice $\mathcal{L}$ there exists a value $q_{c}(\mathcal{L})$ [possibly noninteger; see section 2.1] such that for $q>q_{c}(\mathcal{L})$, the model has exponential decay of correlations at all temperatures including zero, while for $q=q_{c}(\mathcal{L})$ the model has a zero-temperature critical point. Finally, for $q<q_{c}(\mathcal{L})$ any behaviour is possible: often (though not always) the model has a phase transition at nonzero temperature, which may be of either first or second order (e.g., the 3-state model on the triangular [1], or on the diced [45] lattices, respectively). In some other cases, the system has a critical point at $T=0$ and is disordered at any positive temperature, like the AF Ising model $(q=2)$ on the triangular lattice, and in other cases, it is disordered at all temperatures $T \geq 0$, like the AF Ising model on the kagome lattice $[76,77]$. The first task, for any lattice $\mathcal{L}$, is thus to determine the quantity $q_{c}(\mathcal{L})$.

Some AF models at $T=0$ have the remarkable property that they can be mapped exactly onto a 'height' model (in general vector-valued) [22, 27, 43, 67]. Since the height model must either be in a 'smooth' (ordered) or 'rough' (massless) phase, the corresponding zero-temperature spin model must either be ordered or critical, never disordered. Moreover, Henley [22] conjectured that all models that do not admit a height representation are disordered at any temperature $T \geq 0$. When the height model is critical, the long-distance behaviour is that of a massless Gaussian model with some (a priori unknown) 'stiffness tensor' $\mathbf{K}>0$. The critical operators can be 
identified via the height mapping, and the corresponding critical exponents can be predicted in terms of the single parameter $\mathbf{K}$. Height representations thus provide a means for recovering a sort of universality for some (but not all) AF models, and for understanding their critical behaviour in terms of CFT [15]. All the nonuniversal details of the microscopic lattice structure are encoded in the height representation and in the stiffness tensor $\mathbf{K}$. Given these, everything can be understood in terms of the universal behaviour of massless Gaussian fields.

Showing that a given model admits a height representation implies that this is an interesting model, as it has either a zero-temperature critical point, or long-range order at $T=0$, implying that there is a finite-temperature critical point (belonging to some a priori unknown universality class). From now on, we will focus on twodimensional (2D) $q$-state Potts AF models; but there are more models in the literature that admit height representations: the triangular-lattice spin- $s$ Ising antiferromagnet [84] (which is in the smooth phase for $s$ large enough), the 4-state Potts model on the line graph of the square lattice (which is exactly at the roughening transition, but this lattice is not planar) $[42,43]$, a constrained 4-state AF Potts model on the square lattice [10] (which also is in the smooth phase), a special six-vertex model [43], several dimer $[2,3,23,48,63,78]$ and other tiling models $[19,26,38,44]$, and fully-packed loop models $[7,30,40,41,43]$ (see also [27, and references therein]).

Prior to 2009, only a few 2D AF Potts models (i.e., with isotropic nearestneighbour interactions and zero magnetic field) had been studied in detail. The four known models in this class admitting a height representation at $T=0$, also displayed a zero-temperature critical point. These models were $q=2$ on the triangular lattice $[8,74], q=3$ on the square $[10,39,58,67]$ and kagome $[25,43]$ lattices, and $q=4$ on the triangular lattice $[22,56]$. Therefore, by that year, the existence of a zero-temperature critical point could be considered a rather uncommon phenomenon, and no 2D AF Potts model with a height representation and long-range order at $T=0$ was known in the literature.

However, this scenario was challenged in [45]. The authors noted that the very same height representation that was found for the 3-state Potts antiferromagnet on the square lattice, carries over unchanged to any plane quadrangulation. (A plane quadrangulation is a plane graph in which all faces are quadrilaterals or 4-cycles.) Therefore, it was natural to conjecture that $q_{c}=3$ for any plane quadrangulation. This conjecture was tested with the second simplest plane quadrangulation: the diced lattice (i.e., the Laves lattice $[3,6,3,6]$ — using the notation of [20]; see figure 1(b)). Contrary to the previous expectations, it was rigorously proven that this model has long-range order at $T=0$. Furthermore, using high-precision Monte Carlo (MC) simulations, a finite-temperature phase transition was found. The numerical data strongly supports that this critical point belongs to the universality class of the FM 3 -state Potts model. In summary, not only was the naive conjecture mentioned above false, but this work provided also the first example of an 2D AF Potts model with both a height representation and long-range order at $T=0$. In particular, all this implies that $q_{c}$ (diced) $>3$ (numerical estimates from transfer matrices (TM) yield $q_{c}($ diced $\left.) \approx 3.45[34]\right)$.

In a similar way, Moore and Newman [56] observed that the same height repre- 
sentation found for the 4-state Potts AF on the triangular lattice can also be applied without any modification to any Eulerian plane triangulation. (A triangulation is a plane graph in which all faces are triangles; it is Eulerian if moreover all the vertices have even degree.) Again, the natural conjecture is that $q_{c}=4$ for any Eulerian plane triangulation. And again, this conjecture turned out to be false: on one side, there are strong analytic arguments showing that on any Eulerian plane triangulation in which one sublattice consists entirely of vertices of degree 4, the 4-state Potts antiferromagnet has a finite-temperature phase transition [14], so that $q_{c}>4$ for this class of triangulations. In addition, the universality class of this critical point could be predicted from the lattice structure. These findings were tested on two simple Eulerian plane triangulations: the union-jack and the bisected hexagonal lattices (i.e., the Laves $\left[4,8^{2}\right]$ and $[4,6,12]$ lattices, respectively; see [20], [14, figure 2]). In both cases, $\mathrm{MC}$ simulations and TM computations found finite-temperature critical points belonging to the predicted universality classes [14]. In particular, $q_{c}($ union-jack $) \approx 4.33$, and $q_{c}$ (bisected-hexagonal $) \approx 5.40$. This latter result was rather surprising, as it implied not only a finite-temperature critical point for $q=4$, but also for $q=5$. Again, examples of 2D AF $q$-state Potts models were found having a height representation and displaying an ordered phase at low temperature.

As a matter of fact, one can devise plane quadrangulations (e.g., $G_{n}^{\prime}$ and $G_{n}^{\prime \prime}$ ) and plane triangulations (e.g., $H_{n}^{\prime \prime \prime}$ ) such that $q_{c}$ takes arbitrarily large values as $n$ increases [24, figure 2, and tables I and II]. Therefore, there are infinitely many lattices for which there exists a height representation (for $q=3$ or $q=4$ depending whether the lattice is a plane quadrangulation or triangulation, respectively) with an ordered low-temperature phase. All these results imply a qualitative change in the general picture about phase transitions for 2D AF Potts models: the class of these models with a height representation and long-range order at $T=0$ contains actually infinitely many elements.

A natural question is to know whether the class of 2D AF Potts models with a height representation and displaying a critical point at $T=0$ is finite or not. A good stating point is to investigate if there exists any useful condition on the class of plane quadrangulations telling us whether the corresponding 3-state AF Potts model at $T=0$ is critical or ordered. (One could have considered the same question for plane Eulerian triangulations and the 4-state AF Potts model. To our knowledge, this is still an open problem.)

In this paper we shall propose a new criterion, involving graph duality, that appears to distinguish precisely whether the zero-temperature 3-state AF Potts model on a quadrangulation is critical or ordered. (A summary of this work has appeared previously in [51].) This criterion depends on whether the quadrangulation belongs to one of two possible classes. In particular, we will show in section 2.2 , that there is a one-to-one correspondence between plane quadrangulations $\Gamma$ and pairs of dual plane graphs $\left(G, G^{*}\right)$. Then the class of planar quadrangulations can be split into two disjoint subclasses: those of self-dual type, if $G$ is self-dual (i.e., $G \simeq G^{*}$ ); and those of non-self-dual type, otherwise (see definition 2.1).

Using arguments based on the existence of a height representation for these models, we have arrived at the main result of this paper, that can be summarised as 
follows:

Conjecture 1.1 For the three-state antiferromagnetic Potts model on a (periodic) plane quadrangulation $\Gamma$,

(a) If $\Gamma$ is a quadrangulation of self-dual type, then this model has a zero-temperature critical point, so that $q_{c}(\Gamma)=3$. The model is described by a CFT of central charge $c=1$.

(b) If $\Gamma$ is a quadrangulation of non-self-dual type, this model has long-range order at low temperature, and displays a finite-temperature phase transition, so that $q_{c}(\Gamma)>3$. This transition is second-order and lies in the universality class of the 3-state Potts ferromagnet, which is described by a CFT of central charge $4 / 5$.

We have tested this conjecture on four (resp. seven) quadrangulations of self-dual (resp. non-self-dual) type using several complementary techniques: MC simulations, TM computations, and the method of critical polynomials (CP) [28, 35, 36, 70, 71]. These numerical results agree, without exception, with conjecture 1.1. As a side result, we have computed some critical exponent for the 3-state AF Potts model on several quadrangulations of self-dual type not considered before in the literature.

It is worth noticing that there are infinitely many self-dual periodic planar lattices $[4,59,60,69,72,81,85]$. Thus, conjecture 1.1(a) implies that there are also infinitely many 3-state AF Potts models displaying a zero-temperature critical point. This observation entails another qualitative change in the general picture about phase transitions in this kind of models: the class of 2D AF Potts models with a height representation and a zero-temperature critical point also contains infinitely many elements. Finally, there is a close connection between our results and those by Delfino and Tartaglia [13]. They have obtained renormalisation-group (RG) fixed points of CFT invariant under the symmetric group $S_{q}$. The Potts models in conjecture 1.1(a) provide a lattice realisation of their solution I (see [13, table I]).

On the other hand, there are also infinitely many quadrangulations of non-self-dual type (see e.g., the families $G_{n}^{\prime}$ and $G_{n}^{\prime \prime}$ of [24]). Thus, conjecture 1.1(b) implies that there are also infinitely many 3-state AF Potts models displaying long-range order at $T=0$ and undergoing a finite-temperature phase transition. Although this transition has been found in all the cases considered here to be of second order, we cannot rule out the possibility that in some cases it might be of first order (see section 4).

In summary, we have recovered some sort of universality for the 3-state AF Potts model on the whole class of (periodic) plane quadrangulations. The phase diagram of this model will depend only on whether the quadrangulation $\Gamma$ is of self-dual type or not.

As a side result, we have found strong empirical evidence that the Wang-SwendsenKotecký (WSK) algorithm [79,80] for the zero-temperature 3-state AF Potts model on quadrangulations of self-dual type has no critical slowing down (CSD), even though this is a critical point for all these models. In this case, the MC simulations were carried over quadrangulations of this type with toroidal boundary conditions with 
sizes such that the resulting graphs were bipartite. This condition is essential for the WSK Markov-chain to be irreducible (or ergodic) [10,16,52]. The absence of CDS for this algorithm was first observed on the square lattice $[16,67]$.

On the other hand, for the 3-state Potts AF model on five quadrangulations of non-self-dual type, we have found that the WSK algorithm does present CSD. Furthermore, the estimated dynamic critical exponents $z_{\text {int, } \mathcal{M}_{\mathrm{stagg}}^{2}}$ and $z_{\text {int, } \mathcal{M}_{\mathrm{u}}^{2}}$ agree well within errors with the corresponding dynamic critical exponent $z_{\text {int, } \mathcal{M}^{2}}$ for the Swendsen-Wang (SW) algorithm [75] for the FM 3-state Potts model on the square lattice $[18,66]$.

Therefore, we propose the following:

Conjecture 1.2 Consider the Wang-Swendsen-Kotecky algorithm for the three-state antiferromagnetic Potts model on a (periodic) bipartite quadrangulation $\Gamma$ embedded in a torus. Then:

(a) If $\Gamma$ is a quadrangulation of self-dual type, this algorithm has no critical slowing down at the zero-temperature critical point.

(b) If $\Gamma$ is a quadrangulation of non-self-dual type and the finite-temperature phasetransition point of this model is of second order, this algorithm has critical slowing down at the critical temperature. This algorithm belongs to the same dynamic universality class as the Swendsen-Wang algorithm for the 3-state ferromagnetic Potts model.

It is worth noticing that conjecture 1.2(a) provides infinitely many critical models for which the WSK algorithm has no CSD. Before this work (and [51]), the squarelattice case was the only known example with this uncommon (but very desirable) dynamic property. Finally, conjecture 1.2(b) seems 'natural' when compared to conjecture 1.1(b).

This paper is organised as follows. In section 2 we provide the necessary background we need to make this paper as self-contained as possible. Our numerical results are described in section 3. Finally, we discuss the physical interpretation of our numerical findings in section 4. In appendix A, we show how to compute the geometric factor for two of the above quadrangulations.

\section{Basic setup}

This section is devoted to explaining the main definitions we will use afterwards. In particular, in section 2.1, we will review basic facts about the $q$-state Potts model. Section 2.2 will deal with the class of lattices we are interested in; namely, plane quadrangulations. Finally, in section 2.3, we will work out the height representation for the zero-temperature 3-state Potts antiferromagnet on any plane quadrangulation $\Gamma$. This representation will lead us to make some predictions about the critical nature of these Potts models based solely on whether $\Gamma$ is of self-dual type or not. 


\subsection{The Potts model}

The $q$-state Potts model $[62,82,83]$ can be defined on any undirected graph $G=$ $(V, E)$ with vertex set $V$ and edge set $E$. On each vertex of the graph $x \in V$, we place a spin $\sigma_{x} \in \Omega=\{0,1, \ldots, q-1\}$, where $q \geq 2$ is a positive integer. Each spin has $q$ possible values or 'colours', and interacts with those spins located on the neighbouring vertices with some coupling constant $J \in \mathbb{R}$. The partition function of this model is written as

$$
Z_{G}(q, J)=\sum_{\sigma: V \rightarrow \Omega} \exp \left(J \sum_{\{x, y\} \in E} \delta_{\sigma_{x}, \sigma_{y}}\right)
$$

where the first sum is over all possible colourings of the spins of the system, and the second one (inside the exponential) is over all edges of the graph, with $\delta_{\sigma_{x}, \sigma_{y}}$ being the Kronecker delta. The coupling constant is proportional to the inverse of the temperature $T$, and its sign defines the regime of the model: if $J>0$ (resp. $J<0$ ) the model is in the FM (resp. AF) regime.

Even though we will deal with $q=3$ in this paper, it is useful to rewrite (2.1) in the Fortuin-Kasteleyn (FK) representation [17,37]:

$$
Z_{G}(q, v)=\sum_{F \subseteq E} v^{|F|} q^{k(F)},
$$

where the sum is over all spanning subgraphs $(V, F)$ of $G=(V, E), k(F)$ is the number of connected components of $(V, F)$, and the temperature-like parameter $v$ is defined as

$$
v=e^{J}-1
$$

Therefore, in the FM regime $v \geq 0$; in the AF one, $v \in[-1,0)$, and the model is unphysical for $v<-1$ (complex $J$ ). This variable $v$ will be used in the plots shown in section 3. One important consequence of (2.2) is that $Z_{G}$ is a polynomial in both $q$ and $v$, therefore we can promote both variables outside their original physical range. In particular, $q, v$ can be any real, or even complex numbers. This representation gives some sense to the $q$-state Potts model for noninteger values of $q$ : for instance, for the hexagonal lattice, we expect that $q_{c}$ (hexagonal $)=(3+\sqrt{5}) / 2[65$, and references therein].

In Statistical Mechanics one is mainly interested in the thermodynamic limit, in which $G$ is a graph that 'tends to infinity' in a suitable way. In order to achieve this, we first define the free energy per vertex for a finite graph:

$$
f_{G}(q, v)=\frac{1}{|V|} \log Z_{G}(q, v) .
$$

We then define a suitable sequence of graphs $\left(G_{n}\right)$ with $G_{n}=\left(V_{n}, E_{n}\right)$ which is usually a subset of an infinite periodic lattice $\mathcal{G}$ with some boundary conditions (free, cylindrical, toroidal, etc). The thermodynamic limit of the free energy is defined as the limit

$$
f(\mathcal{G} ; q, v)=\lim _{n \rightarrow \infty} \frac{1}{\left|V_{n}\right|} \log Z_{G_{n}}(q, v)
$$


if that limit exists. It is well known that for $q \in \mathbb{N}$ and $v>-1$ [using the spin representation (2.1)] or for $q>0, v \geq 0$ [using the FK representation (2.2)] this limit exists, and it is a continuous function of $v$; but it might fail to be analytic at some points (where phase transitions occur). For other values of the parameters $q, v$ the very existence and/or uniqueness of the above limit is a difficult question.

\subsection{Plane quadrangulations}

In this section we will describe how to build a general plane quadrangulation. This construction was already briefly outlined in [14].

For any (finite or infinite) graph $G=(V, E)$ embedded in the plane, the dual graph $G^{*}=\left(V^{*}, E^{*}\right)$ is defined by placing a vertex in each face of $G$ and drawing an edge $e^{*}$ across each edge $e$ of $G$. Since $G^{* *}=G$, we refer to the pair $\left(G, G^{*}\right)$ as a dual pair. A graph $G$ is called self-dual if $G$ is isomorphic to $G^{*}$.

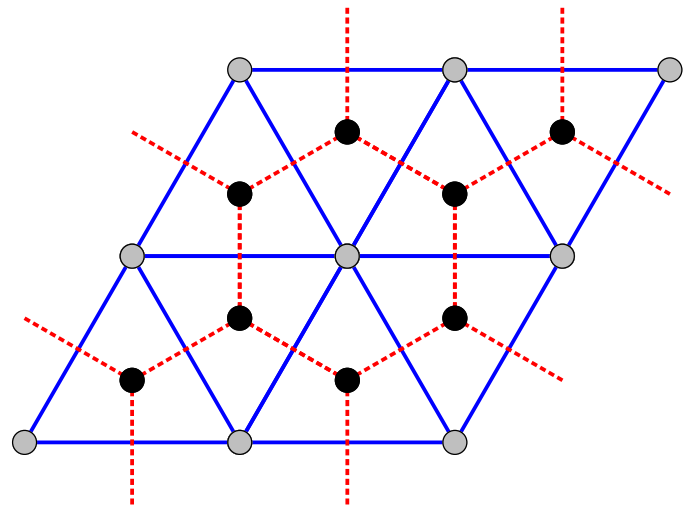

(a)

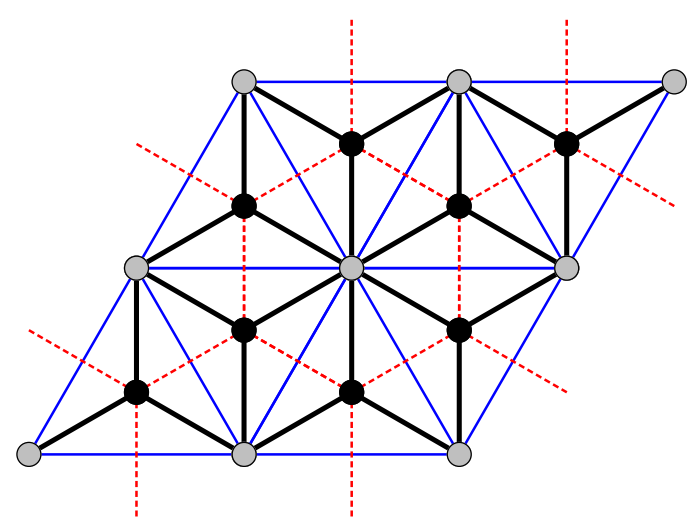

(b)

Figure 1: Building the diced lattice from the dual pair $\left(G_{0}, G_{1}\right)=$ (triangular, hexagonal). (a) We show a triangular lattice $G_{0}=\left(V_{0}, E_{0}\right)$ with gray vertices and solid (blue) edges. We also depict its dual hexagonal lattice $G_{1}=\left(V_{1}, E_{1}\right)=G_{0}^{*}$ with black vertices and dashed (red) edges. (b) We show the corresponding quadrangulation $\Gamma=\mathcal{Q}\left(G_{0}\right)=$ diced lattice, with vertex set $V_{0} \cup V_{1}$ (depicted as black and gray vertices), and edge set depicted as solid thick black lines. [The edges of the underlying triangular and hexagonal lattices are also depicted as in panel (a). They are the diagonals of the quadrangles.]

Let us consider a connected plane graph $G=(V, E)$ and its dual graph $G^{*}=$ $\left(V^{*}, E^{*}\right)$. See figure 1 (a) for $G=$ triangular lattice and $G^{*}=$ hexagonal lattice (see [14, figure 1] for a more general example). We then define the new graph $\Gamma=$ $\left(V \cup V^{*}, E^{\prime}\right)$, such that its vertex set is $V \cup V^{*}$, and its edge set $E^{\prime}$ contains all edges $e=\{x, y\} \in E^{\prime}$, whenever $x \in V$ belongs to the boundary of the face of $G$ that contains the dual vertex $y \in V^{*}$ (see figure $1(\mathrm{~b})$ for $\Gamma=$ diced lattice). This new graph $\Gamma$ is a plane quadrangulation: each face of $\Gamma$ contains exactly one pair of 
diametrically opposite vertices of $V$, corresponding to an edge $e \in E$; and another pair of diametrically opposite vertices of $V^{*}$, which correspond to the dual edge $e^{*} \in E^{*}$. As a matter of fact, $\Gamma$ is the dual of the medial graph $\mathcal{M}(G)=\mathcal{M}\left(G^{*}\right)$. (In particular, the diced lattice of figure $1(\mathrm{~b})$ is the dual of the kagome lattice, which is the medial graph of either the triangular and the hexagonal lattice.)

Indeed, there is a one-to-one correspondence between plane quadrangulations $\Gamma$ and dual pairs of plane graphs $\left(G_{0}, G_{1}\right)$. If we consider a connected plane quadrangulation $\Gamma=(V, E)$, then $\Gamma$ is bipartite, and therefore $V=V_{0} \cup V_{1}$. We can define two sublattices $G_{0}=\left(V_{0}, E_{0}\right)$ and $G_{1}=\left(V_{1}, E_{1}\right)$ by drawing edges across the diagonals of the quadrilateral faces, as in figure $1(\mathrm{~b})$. Those edges connecting vertices of $V_{0}$ belong to $E_{0}$, and conversely, those connecting vertices of $V_{1}$ belong to $E_{1}$. Finally, it is easy to see that $G_{0}$ and $G_{1}$ form a dual pair.

Finally, as $G_{0}$ and $G_{1}$ play the same role in the above constructions, both pairs $\left(G_{0}, G_{1}\right)$ and $\left(G_{1}, G_{0}\right)$ lead to the same quadrangulation $\Gamma$. For simplicity, we will denote the quadrangulation arising from the dual pair $\left(G_{0}, G_{1}\right)$ as $\mathcal{Q}\left(G_{0}\right)=\mathcal{Q}\left(G_{1}\right)$. Let us formalise these ideas in the following:

Definition 2.1 The plane quadrangulation $\Gamma=\mathcal{Q}\left(G_{0}\right)$ associated to the dual pair $\left(G_{0}, G_{1}\right)$ is of self-dual type if $G_{0}$ is self-dual, and of non-self-dual type otherwise.

For instance, the square lattice $[=\mathcal{Q}$ (square) $]$ is a quadrangulation of self-dual type (both $G_{0}$ and $G_{1}$ are themselves square lattices), while the diced lattice [= $\mathcal{Q}$ (triangular)] is a quadrangulation of non-self-dual type (the sublattices are the triangular and hexagonal lattices).

In this paper, we will restrict attention to periodic plane lattices. It is well known (and obvious) that the square lattice is self-dual; what seems to be less well known is that there exist infinitely many self-dual periodic plane lattices $[4,59,60,69,72$, 81,85], including the 'hextri' [59, figures 1 and 10] [72, figure 16] [81, figure 1b], the 'house' [59, figure 2], the martini-B [69, figure 8], and the 'cmm-pmm' [72, figure 29] lattices. In particular, from each of these lattices we can construct the corresponding quadrangulation of self-dual type. Those are depicted in figure 2. This figure shows that all these quadrangulation of self-dual type $\Gamma$ can be regarded as a square Bravais lattice $\mathcal{B}_{\Gamma}$ with a basis formed by $n_{\Gamma}$ vertices. In particular, $n_{\mathcal{Q} \text { (hextri) }}=6, n_{\mathcal{Q} \text { (house) }}=$ $n_{\mathcal{Q}(\text { martini-B })}=8$, and $n_{\mathcal{Q}(\mathrm{cmm}-\mathrm{pmm})}=24$. We say that a quadrangulation $\Gamma$ has size $L \times N$ when the underlying square Bravais lattice $\mathcal{B}_{\Gamma}$ has size $L \times N$.

In addition, we will consider seven quadrangulations of non-self-dual type:

- $\mathcal{Q}($ diced $)=$ dual ruby. This quadrangulation is the Laves lattice $[3,4,6,4]$; see [20, figure 2.7.1].

- $\mathcal{Q}$ (martini), where the martini lattice is depicted in [69, figure 1]. This is a 2-homeohedral tiling of valence 3 in the notation of ref. [20, p. 186]: this is a cubic graph with vertices of type $\left(3,9^{2}\right)$ and $\left(9^{3}\right)$ in the notation of ref. [20].

- $\mathcal{Q}$ (ruby), where the ruby lattice is the Archimedean lattice $(3,4,6,4)[20$, figure 2.1.5]. 
- $\mathcal{Q}($ asanoha $)$, where the asanoha lattice is the Laves lattice $\left[3,12^{2}\right]$; see $[20$, figure 2.7.1].

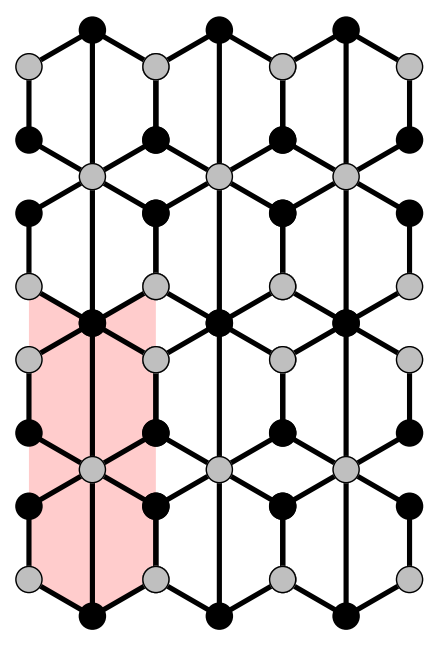

(a)

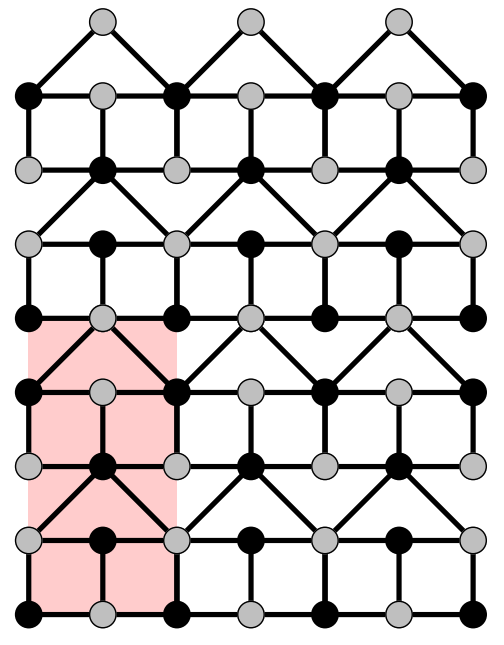

(b)

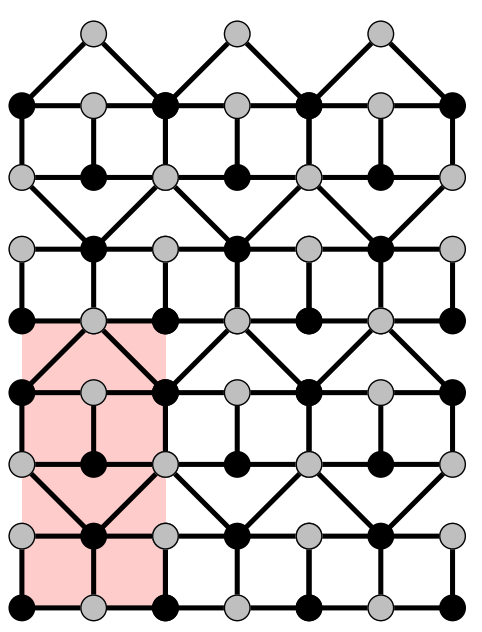

(c)

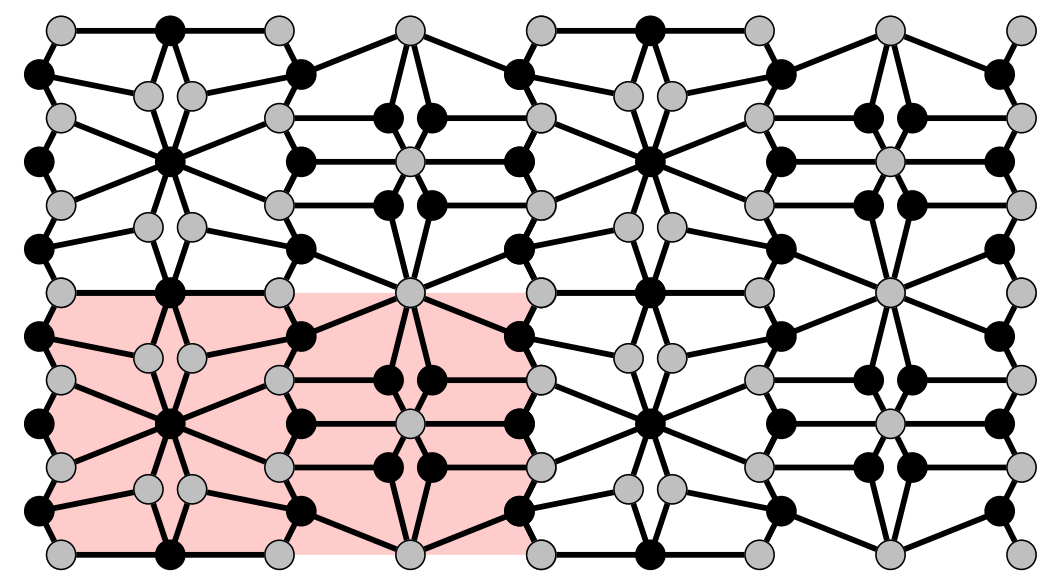

(d)

Figure 2: The four plane quadrangulations of self-dual type considered in this paper: (a) $\mathcal{Q}$ (hextri), (b) $\mathcal{Q}$ (house), (c) $\mathcal{Q}$ (martini-B), and (d) $\mathcal{Q}$ (cmm-pmm). The pink area in each panel shows the corresponding unit cell. We have depicted finite pieces of such lattices with free boundary conditions and sizes $3 \times 2$ (unit cells), except for (d) which has size $2 \times 2$ (see text).

- $\mathcal{Q}$ (cross), where the cross lattice is the Archimedean lattice $(4,6,12)$. The dual cross lattice is also called the bisected hexagonal lattice [14, figure 2(b)] and corresponds to the Laves lattice [4,6,12]; see [20, figure 2.7.1]. 
- $G_{2}^{\prime \prime}=\mathcal{Q}$ (union-jack) [14, figure $\left.2(\mathrm{a})\right]$, where the union-jack lattice is the Laves lattice $\left[4,8^{2}\right]$ (see $[20$, figure 2.7.1]), and the dual of the four-eight lattice.

- $G_{3}^{\prime \prime}=\mathcal{Q}$ (decorated four-eight) [24, figure 2, central panel]. The four-eight lattice is the Archimedean lattice $\left(4,8^{2}\right)$ [20, figure 2.1.5]. The decorated four-eight lattice is obtained from the former one by subdividing once those edges shared by two octogonal faces. Its dual is the lattice $G_{2}^{\prime}$ [24, figure 2, left panel].

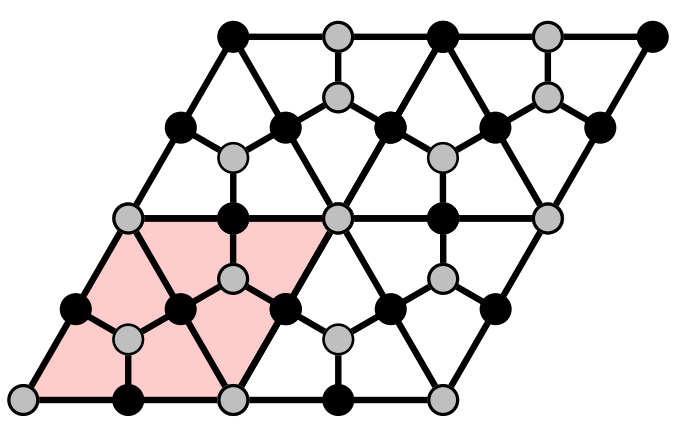

(a)

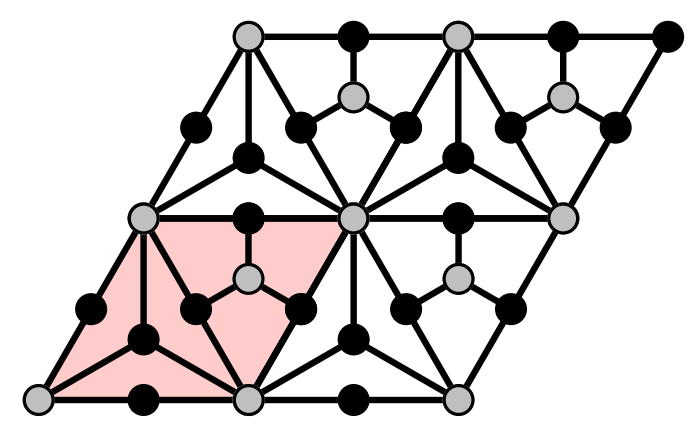

(b)

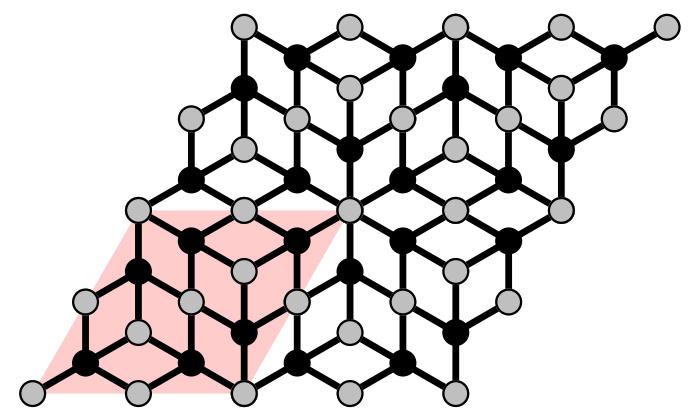

(c)

Figure 3: Three quadrangulations of non-self-dual type considered in this paper with an underlying triangular Bravais lattice: (a) $\mathcal{Q}$ (diced) = dual ruby, (b) $\mathcal{Q}$ (martini), and (c) $\mathcal{Q}$ (ruby). The pink areas show the corresponding unit cells. We have depicted finite pieces of such lattices with free boundary conditions and sizes $2 \times 2$ (unit cells).

Notice that this list encompasses several qualitatively distinct cases: either $G_{0}$ or $G_{1}$ is a plane triangulation (i.e., asanoha, dual cross, dual martini, and union-jack), or a plane quadrangulation (i.e., diced, dual ruby, and $G_{2}^{\prime}$ ). These seven quadrangulations are depicted in figures 3-4.

Concerning their translational invariance, there are five lattices with an underlying triangular Bravais lattice with a basis formed by $n_{\mathcal{Q} \text { (diced) }}=n_{\mathcal{Q} \text { (martini) }}=6$, $n_{\mathcal{Q} \text { (asanoha) }}=9, n_{\mathcal{Q} \text { (ruby) }}=12$, and $n_{\mathcal{Q} \text { (cross) }}=18$. The other two quadrangulations have an underlying square Bravais lattice with a basis formed by $n_{G_{2}^{\prime \prime}}=6$, and 


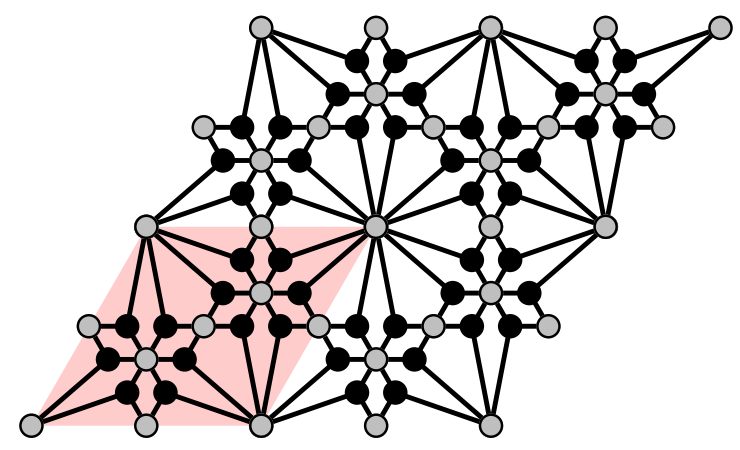

(a)

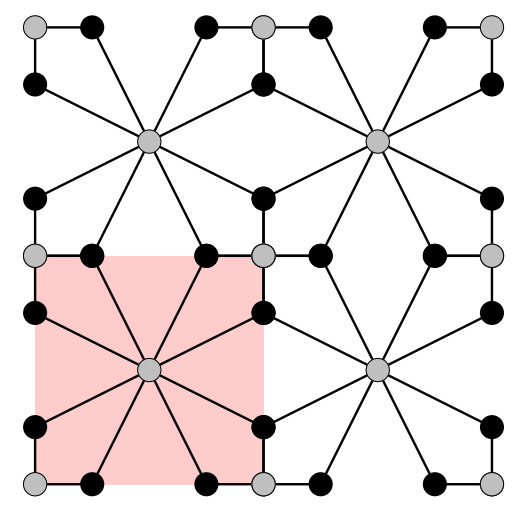

(c)

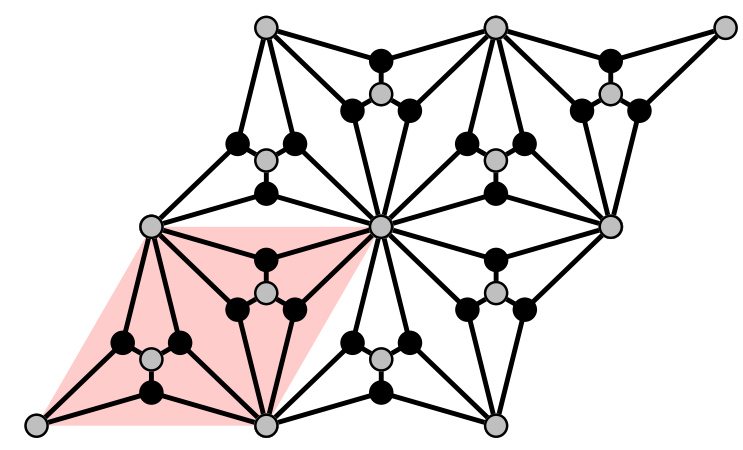

(b)

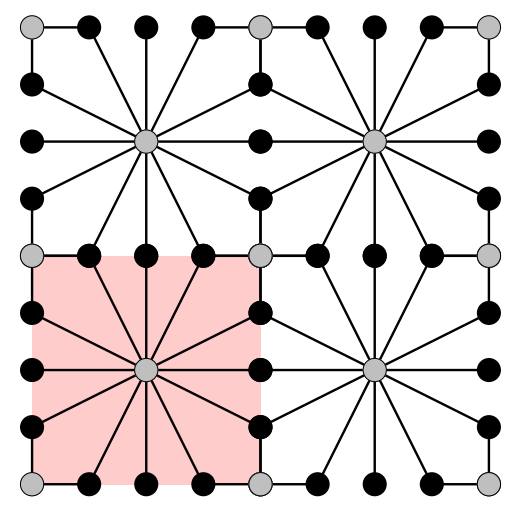

(d)

Figure 4: Quadrangulations of non-self-dual type considered in this paper. We show two of them with an underlying triangular Bravais lattice: (a) $\mathcal{Q}$ (cross), and (b) $\mathcal{Q}$ (asanoha). We also show another two quadrangulations with an underlying square Bravais lattice: (c) $G_{2}^{\prime \prime}$, and (d) $G_{3}^{\prime \prime}$. The lattices are depicted as in figure 3 .

$n_{G_{3}^{\prime \prime}}=8$. Again, we say that a quadrangulation $\Gamma$ has size $L \times N$ when the corresponding underlying square Bravais lattice $\mathcal{B}_{\Gamma}$ has size $L \times N$.

Remark. The above construction of a plane quadrangulation $\Gamma$ associated to a pair of dual plane graphs $\left(G_{0}, G_{1}\right)$ can be extended to any closed orientable surface. If $G_{0}$ is a finite connected graph embedded in a closed orientable surface $S$, then one can construct its dual in an analogous way, so that the dual graph $G_{1}$ is also embedded in $S$. Then, the quadrangulation $\Gamma$ can be built as before, and it is also a graph embedded in $S$. In this way, for any dual pair $\left(G_{0}, G_{1}\right)$ there is a unique quadrangulation $\Gamma$ embedded in the same surface. However, the inverse relation is false: there are quadrangulations in a closed orientable surface that do not correspond to a dual pair. Simple examples of these quadrangulations are square lattices embedded in a torus with sizes $(2 L+1) \times 2 N, 2 L \times(2 N+1)$ and $(2 L+1) \times(2 N+1)$ for any $L, N \in \mathbb{N}$. The 
reason is that these graphs are not bipartite. This observation leads to the following statement: there is a one-to-one correspondence between bipartite quadrangulations embedded in a closed orientable surface $S$ and dual pairs $\left(G_{0}, G_{1}\right)$ embedded also in $S$. We will be interested in section 3.1 in bipartite quadrangulations embedded in a torus.

\subsection{Height representation}

In this section we will discuss the height representation for the AF 3-state Potts model on a quadrangulation. In particular, we will extend the well-known height representation for the square lattice $[10,58,67]$ to a general plane quadrangulation. This extension is not however, the most general case discussed in $[22,43,67]$. We refrain from repeating this general setup here to keep the exposition short.

We consider a finite subset of a plane quadrangulation $\Gamma=(V, E)$ with free boundary conditions (to ensure that everything is well defined). This quadrangulation is associated to the dual pair $\left(G_{0}, G_{1}\right)$, as explained in the previous section with $G_{0}=\left(V_{0}, E_{0}\right)$ and $G_{1}=\left(V_{1}, E_{1}\right)$. We define the zero-temperature 3-state AF Potts model as usual: to each vertex $x \in V$, we assign a spin variable $\sigma_{x} \in\{0,1,2\}$, such that two neighbouring spins cannot have the same value: i.e., if $\{x, y\} \in E$, then $\sigma_{x} \neq \sigma_{y}$. Indeed, as $\Gamma$ is bipartite, there are many possible ground states (= proper colourings of $\Gamma$ ) without frustration.

The first step consists in defining a height rule, so we can map the spin configuration to a height configuration. In our case, the microscopic height variables $h(x)$ live on the vertices of $\Gamma$, and they are defined as follows:

- Pick, without loss of generality, any vertex $0 \in V_{0}$. We will call this distinguished vertex the 'origin'. We fix the height at the origin as $h(0) \equiv 0,4,2(\bmod 6)$ according to whether $\sigma_{0}=0,1,2$. This choice ensures that

$$
h(0) \equiv \sigma_{0} \quad(\bmod 3), \quad h(0) \equiv 0 \quad(\bmod 2) .
$$

- The increment in height in going from vertex $x$ to a neighbour vertex $y$ is given by

$$
h(x)-h(y) \equiv \sigma_{x}-\sigma_{y}(\bmod 3), \quad h(x)-h(y)= \pm 1
$$

This rule is well defined, as the change $\Delta h$ around any quadrilateral face is zero. (If four numbers \pm 1 add up to $0(\bmod 3)$, they must be two +1 and two -1 , so their sum is zero.) The above rules $(2.6) /(2.7)$ fix the height $h(x) \bmod 6$ at any vertex $x \in V=V_{0} \cup V_{1}$ :

$$
h(x) \equiv \sigma_{x} \quad(\bmod 3), \quad h(x) \equiv\left\{\begin{array}{lll}
0 & (\bmod 2) & \text { if } x \in V_{0} \\
1 & (\bmod 2) & \text { if } x \in V_{1}
\end{array}\right.
$$

once we know the height at the origin $h(0)$, and the sublattice $x$ belongs to. Then the height lattice (i.e., the subset of $\mathbb{R}^{D}$ where the height variables live) is $\mathbb{Z}$ with $D=1$. 
The second step is to determine the ideal states: they are in general families of proper colourings of $\Gamma$ whose corresponding height configurations are macroscopically flat and maximize the entropy density. These ideal states depend on the type of quadrangulation we are considering:

- If $\Gamma$ is a plane quadrangulation of self-dual type, then both sublattices are equivalent $G_{0} \simeq G_{1}$. Therefore, there are six ideal states such that one sublattice is ordered (i.e, all spins take a constant value), while the other sublattice is disordered (i.e., the spins take randomly the other two values). The system has to choose which sublattice is the ordered one, and then it has to choose which value is taken by all the spins in such sublattice. If $\Gamma$ is associated to the dual pair $\left(G_{0}, G_{1}\right)$ we will label our ideal states as $a / b c($ resp. $a b / c)$ with $a, b, c=0,1,2$, if the $G_{0}$ (resp. $G_{1}$ ) sublattice is ordered.

- If $\Gamma$ is a plane quadrangulation of non-self-dual type, then $G_{0}$ and $G_{1}$ are no longer equivalent $\left(G_{0} \neq G_{1}\right)$. Let us first consider the most often case, where one sublattice has less vertices (or a smaller vertex density) than the other one. Without loss of generality, if we assume that $\left|V_{0}\right|<\left|V_{1}\right|$, then the ordered sublattice will be $G_{0}$, and the disordered one will be $G_{1}$. Therefore, we expect three ideal states of the form $a / b c$. The case for which $\left|V_{0}\right|=\left|V_{1}\right|$ needs more work, and will be considered at the end of this section.

In both cases, these ideal states are macroscopically flat: the microscopic height at the vertices of the ordered sublattice is constant $h_{\circ}$, and on the vertices of the other sublattices, the height takes randomly the values $h_{\circ} \pm 1$. We can therefore label each ideal state by its average height $\langle h(x)\rangle=h_{\circ}$.

The above ideal states also maximize the zero-temperature entropy density $W(\Gamma ; q)$ in the thermodynamic limit (see (2.5)):

$$
W(\Gamma ; q)=e^{f(\Gamma ; q,-1)}=\lim _{n \rightarrow \infty} Z_{\Gamma_{n}}(q,-1)^{1 /\left|U_{n}\right|},
$$

for a suitable sequence $\left(\Gamma_{n}\right)$ of graphs $\Gamma_{n}=\left(U_{n}, F_{n}\right)$ tending, as $n \rightarrow \infty$, to some infinite periodic quadrangulation $\Gamma$. We can compare the entropy of ideal states to the entropy of three-colourings tout court:

- Let $\Gamma_{n}=\left(V_{n}, E_{n}\right)$ be a plane quadrangulation of self-dual type with $\left|V_{n}\right|=n$. The ideal-state entropy density is given by

$$
W(\Gamma ; 3)=\lim _{n \rightarrow \infty}\left(6 \times 2^{n / 2}\right)^{1 / n}=\sqrt{2} \approx 1.41421 .
$$

This result is not far from (and smaller than) Lieb's exact result for the square lattice $W($ square, 3$)=(4 / 3)^{3 / 2} \approx 1.53960[49,50]$.

- Let $\Gamma_{n}=\left(V_{n}, E_{n}\right)$ be a plane quadrangulation of non-self-dual type, with $\left|V_{n}\right|=$ $n$, and associated to the dual pair $\left(G_{0, n}, G_{1, n}\right)$ with $G_{j, n}=\left(V_{j, n}, E_{j, n}\right)$ for $j=$ 0,1 , and $\left|V_{0, n}\right|+\left|V_{1, n}\right|=n$. The ideal-state entropy density is given by

$$
W(\Gamma ; 3)=\lim _{n \rightarrow \infty}\left(3 \times 2^{\left|V_{1, n}\right|}\right)^{1 / n}=\lim _{n \rightarrow \infty} 2^{\left|V_{1, n}\right| / n} .
$$


In the diced case, $\left|V_{0, n}\right|=n / 3$ and $\left|V_{1, n}\right|=2 n / 3$, so $W($ diced, 3$)=2^{2 / 3} \approx$ 1.5874. This value is close to (and smaller than) the estimated value by Chen et al. $[12]: W($ diced, 3$) \approx e^{0.473839} \approx 1.6062$.

The examples discussed above show that the naive ideal-state entropy density is slightly smaller that the true entropy density for the square and diced lattices, respectively. But this ideal-state picture captures the relevant physics of the corresponding models, as we shall see below.

It follows from (2.8) that there is a one-to-one correspondence between ideal states and heights mod 6. The ideal-state lattice $\mathcal{I}$ (which is the set of all average heights of ideal states) for the quadrangulations of self-dual type is $\mathcal{I}_{\text {sf }}=\mathbb{Z}$; but for quadrangulations of non-self-dual type is $\mathcal{I}_{\text {nsf }}=2 \mathbb{Z}$ (see figure 5).

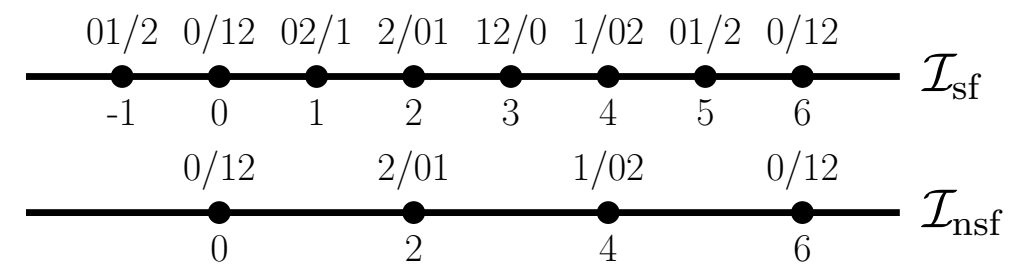

Figure 5: Ideal-state lattice for the zero-temperature 3-state AF Potts model on a plane quadrangulation of self-dual type (top figure labeled $\mathcal{I}_{\text {sf }}$ ), and for the same model on a quadrangulation of non-self-dual type (bottom figure labeled $\mathcal{I}_{\text {nsf }}$ ). The dots depict the ideal states: the numbers below (resp. above) each dot represent the average height $h_{\circ}$ (resp. the spin structure) of the corresponding ideal state (see text).

Notice that a given ideal state corresponds to infinitely many different average heights $h \in \mathcal{I}$. In particular, there is a one-to-one correspondence between ideal states and the $\operatorname{coset} \mathcal{I} / \mathcal{R}$, where $\mathcal{R}$ is the repeat lattice. This is a subgroup of $\mathbb{Z}$ such that if $h \in \mathcal{I}$ is a given ideal state, then $h+\mathcal{R}$ corresponds to the same ideal state. In our case, it is obvious that $\mathcal{R}=6 \mathbb{Z}$ for any plane quadrangulation, as the heights are defined modulo 6 (see figure 5 ).

Up to this point the arguments are rigorous. Now we guess that typical configurations of the spin model are built from relatively large domains (where on each of these domains, the spin configuration corresponds to small fluctuations around one of the above ideal states), separated by relatively narrow interfaces. If we then define suitable coarse-grained height variables $\bar{h}(x)$, we expect that they will take values in or near the ideal-state lattice $\mathcal{I}$, except at the boundary between domains. The long-wavelength behaviour of such a coarse-grained model is believed to be controlled by the effective coarse-grained Hamiltonian (or Euclidean action)

$$
H=\int\left[\frac{K}{2}|\nabla \bar{h}(x)|^{2}+V_{\text {lock }}(\bar{h}(x))\right] \mathrm{d}^{2} x,
$$


where we have taken into account that $D=1$. The first term in the integral takes into account the entropy of small fluctuations around the ideal states; and the locking potential $V_{\text {lock }}$ favours the heights to take their values in $\mathcal{I}$. The coupling constant (or stiffness) $K$ controls the physics of this model: there is a critical value $K_{c}$ such that if $K<K_{c}$ (resp. $K>K_{c}$ ), $V_{\text {lock }}$ is irrelevant (resp. relevant) in the RG sense. Therefore, if $K<K_{c}$ the height model is in the rough phase, and its long-wavelength behaviour is described by a massless Gaussian model with height correlations diverging logarithmically with distance. In this case, the corresponding spin model is critical, and it is described by a CFT with central charge $c=1$. On the contrary, if $K>K_{c}$, the height model is in its smooth phase, exhibiting long-range order and bounded fluctuations around the ordered state. Then, the corresponding spin model describes small fluctuations around one of the ideal states. At $K=K_{c}$ the system is at the roughening transition, and the spin system is also critical.

If $K<K_{c}$ this approach also predicts the number of critical observables and their corresponding critical exponents in terms of the unique free parameter $K$. In particular, the correlation functions of local operators of the coarse-grained heights should have the periodicity of the repeat lattice $\mathcal{R}$. Therefore, the Fourier transform of such correlators should contain only wavevectors belonging to the reciprocal of the repeat lattice

$$
\mathcal{R}^{o}=\{G \in \mathbb{R}: G \cdot a \in 2 \pi \mathbb{Z}, \forall a \in \mathcal{R}\} .
$$

In particular, we have three candidates for critical observables in this model

- The staggered magnetisation with $G= \pm \pi / 3$.

- The uniform magnetisation with $G= \pm 2 \pi / 3$.

- The staggered polarisation with $G= \pm \pi$ (see [10,67] for details).

Given the wavelength $G$, the corresponding correlator will decrease algebraically with distance like $\sim|x|^{-\eta_{G}}$ with

$$
\eta_{G}=\frac{G^{2}}{2 \pi K}
$$

In particular, all critical exponents depend on the single parameter $K$. The usual critical-exponents ratios can be computed by using (2.14) and the usual scaling relation

$$
\left.\frac{\gamma}{\nu}\right|_{G}=2-\eta_{G}=2-\frac{G^{2}}{2 \pi K} .
$$

Notice that the value of $K_{c}$ is obtained by making the locking potential exactly marginal: i.e., $\eta=4$. This potential has the periodicity of the ideal-state lattice $\mathcal{I}$, so its Fourier transform can only contain wavevectors $G$ belonging to the reciprocal of the ideal-state lattice $\mathcal{I}^{\circ}$. Then the critical stiffness $K_{c}$ is given by

$$
K_{c}=\frac{a_{\mathcal{I}^{\circ}}^{2}}{8 \pi}= \begin{cases}\frac{\pi}{2} & \text { if } \Gamma \text { is of self-dual type } \\ \frac{\pi}{8} & \text { if } \Gamma \text { is of non-self-dual type. }\end{cases}
$$


as $\mathcal{I}_{\text {sf }}^{\circ}=2 \pi \mathbb{Z}$ for quadrangulations of self-dual type, and $\mathcal{I}_{\text {nsf }}^{\circ}=\pi \mathbb{Z}$ for quadrangulations of non-self-dual type. Note that the critical stiffness for the latter case is four times smaller than for quadrangulations of self-dual type.

Let us now discuss the behavior of the 3-state AF Potts model on a plane quadrangulation depending of its type. This discussion will lead naturally to conjecture 1.1 stated in the Introduction.

\section{Quadrangulations of self-dual type}

In the square-lattice case, from the known result for the staggered magnetisation $\eta_{\text {stag }}=1 / 3[58,61]$, the value $K=\pi / 6$ follows from $(2.14)$, and the other exponents for this model can be computed by using $(2.14) /(2.15)$. Notice that in this case, $K<K_{c}=\pi / 2$ (2.16). Therefore this spin model has a zero-temperature critical point whose large-distance behaviour is governed by a CFT with central charge $c=1$. In addition, the three observables mentioned above are relevant; although only the first two have a diverging susceptibility.

We expect that for the 3-state AF Potts model on any plane quadrangulation of self-dual type all the above conclusions should hold by using standard universality arguments: the symmetry and ground-state degeneracy are exactly the same (i.e., six ideal states, each of them with a sublattice ferromagnetically ordered). Moreover, the ideal-state and repeat lattices are identical to the square-lattice case. Therefore, $q_{c}(\Gamma)=3$ for any quadrangulation $\Gamma$ of self-dual type. However, as there is a free parameter $K$, the critical exponents may be (and in fact are) lattice dependent through the unique free parameter $K$. Therefore, the stiffness $K$ encodes the microscopic properties of the lattice for this class of models.

In this sense, we recover some sort of universality for the whole family of plane periodic quadrangulations of self-dual type. The only difference is that the critical exponents depend on $K$, rather than being the same for all elements of this family. This discussion motivates part (a) of conjecture 1.1.

\section{Quadrangulations of non-self-dual type}

If $\Gamma$ is of non-self-dual type, then the two sublattices are not equivalent. Let us assume in this preliminary discussion that there is one sublattice $\left(G_{0}\right)$ that contains less vertices than the other one $\left(G_{1}\right)$ (or, equivalently, that the fraction of vertices belonging to $G_{0}$ is smaller than that of $G_{1}$ ). Then, as we discussed earlier, all the spins on $G_{0}$ will take the same value, while those on $G_{1}$ will take randomly the other two values. Therefore, we have only three ideal states (each of them with $G_{0}$ ferromagnetically ordered).

The diced lattice [45] [depicted in figure 1(b)] is the simplest case: the triangular sublattice $G_{0}$ contains one third of the vertices, and each of them has degree six, while $G_{1}$ is an hexagonal sublattice with two thirds of the vertices, each of them of degree three. (The average degree is four, as it should be.) Then $G_{0}$ is the ordered sublattice in the three ideal states. In fact, it has been rigorously proven that the 3 -state Potts antiferromagnet has long-range order at $T=0$ [45] with three ordered 
states (one for each ideal state). Furthermore, it has been shown numerically via MC simulations, that there is a finite-temperature critical point belonging to the 3-state $F M$ Potts-model universality class.

If we consider the 3-state AF Potts model on any plane periodic quadrangulation of non-self-dual type, we find that the symmetry and ground-state degeneracy are the same as for the diced lattice. Therefore, using the same universality arguments as for the self-dual case, we expect that the ground state is ordered with three distinct and coexisting phases. Therefore, there should be a phase-transition at some (latticedependent) finite temperature $v_{c}(\Gamma)$. The existence of such a transition implies that $q_{c}(\Gamma)>3$ for any quadrangulation $\Gamma$ belonging to this family.

The fact that the critical stiffness $K_{c}=\pi / 8 \approx 0.39269908$ (2.16) is four times smaller than for the self-dual case, implies that for the non-self-dual case, it is, in principle, more difficult to fall in the critical case $K<K_{c}=\pi / 8$. In particular, the staggered polarisation operator mentioned above has wavevector $G= \pm \pi$, which coincides with the wavevector of the locking potential; therefore it is no longer a relevant operator, but a marginal one. (Actually, the stiffness for all quadrangulations of self-dual type displayed in table 1 are larger than this critical value.)

The nature of the predicted finite-temperature phase transition is a priori not clear: it might be either a first- or a second-order critical point. Again, if this finitetemperature phase transition is of second order, then universality predicts that it must belong to the universality class of the 3 -state FM Potts model. (At $T=0$, the ground-state of both models has the same $\mathbb{Z}_{3}$ symmetry and degeneracy.) Moreover, the previous results for the diced lattice and the new ones for the other seven lattices considered in this paper agree well with this scenario. This discussion motivates part (b) of conjecture 1.1.

Remark. The above arguments assume that the quadrangulation $\Gamma$ corresponds to a dual pair $\left(G_{0}, G_{1}\right)$ such that $\left|V_{0}\right|<\left|V_{1}\right|$. However, there are quadrangulations of non-self-dual type that satisfy $\left|V_{0}\right|=\left|V_{1}\right|$ [e.g. $\Gamma=\mathcal{Q}$ (diced) and $\mathcal{Q}$ (ruby)]. In these cases, there is no a priori reason why only three out of the initial six candidates are actually the ideal states of the system.

If one of the sublattices (say, $G_{0}$ ) has a sub-sublattice $G_{0,0}$ with vertices with a degree larger than the degrees of the vertices on the other sub-sublattices of $G_{0}$ and $G_{1}$, then we expect that $G_{0}$ should be the 'ordered' lattice. In fact, the numerical study of $\mathcal{Q}$ (diced) and $\mathcal{Q}$ (ruby) provides additional support to this argument: the sublattice $G_{0}$ that is more ordered is indeed the one containing the vertices with larger degree. However, in this case the sublattice $G_{0}$ and its sub-sublattice $G_{0,0}$ are not completely ordered, contrary to what happened to $G_{0}$ when $\left|V_{0}\right|<\left|V_{1}\right|$.

In other words, even though the 'naive' entropy density of the two sets of ideal states is the same, the true entropy density is not, as one has to consider also the fluctuations around these ideal states. And this true entropy density is larger for the sublattice containing a sub-sublattice with vertices of larger degree.

Finally, notice that if we have a quadrangulation of non-self-dual type $\Gamma$ associated to a dual pair $\left(G_{0}, G_{1}\right)$ satisfying $\left|V_{0}\right|=\left|V_{1}\right|$, then the quadrangulation $\mathcal{Q}(\Gamma)$ associated to a new dual pair $\left(G_{0}^{\prime}, G_{1}^{\prime}\right)$ will also satisfy $\left|V_{0}^{\prime}\right|=\left|V_{1}^{\prime}\right|$. This observation uses 
the fact that the average degree on a quadrangulation is 4, and provides additional examples to further test this subtle point in our arguments.

\section{Numerical results}

In this section we will describe our numerical tests to confirm or disprove conjectures 1.1 and 1.2. We will consider the four quadrangulations of self-dual type shown in figure 2 (namely, $\mathcal{Q}$ (hextri), $\mathcal{Q}$ (house), $\mathcal{Q}$ (martini-B), and $\mathcal{Q}(\mathrm{cmm}-\mathrm{pmm})$ ), and the seven quadrangulations of non-self-dual type shown in figures 3-4 (namely, $\mathcal{Q}$ (diced), $\mathcal{Q}$ (martini), $\mathcal{Q}$ (ruby), $\mathcal{Q}$ (asanoha), $\mathcal{Q}$ (cross), $G_{2}^{\prime \prime}$ and $\left.G_{3}^{\prime \prime}\right)$. In addition, we also have two already well-known cases: the square [10,67] and the diced [45] lattices.

Most of our numerical work consist in high-precision MC simulations of the 3-state Potts AF model on these seven lattices; but we will also use other techniques: TM and CP. All these methods will be described in the following sections.

\subsection{Monte-Carlo simulations}

We have made extensive MC simulations for the eleven lattices mentioned above using the Wang-Swendsen-Kotecký (WSK) cluster algorithm [79,80]. To avoid surface effects, we have considered the Potts model on finite subsets of each lattice with periodic boundary conditions. Although WSK is irreducible (or ergodic) on any graph $G$ at any non-zero temperature, periodic boundary conditions may cause troubles with the ergodicity of this algorithm at zero temperature. In general, at $T=0$, the Potts-model probability distribution becomes the uniform measure over the set of proper q-colourings of $G$. While for planar graphs, ergodicity of WSK can be proven for any $q>\chi(G)$, where $\chi(G)$ is the chromatic number of $G$ [52, Corollary 4.5], for non-planar graphs the ergodicity of WSK can be guaranteed only for $q \geq \Delta+1$, where $\Delta$ is the maximum degree of $G$ [52, Corollary 2.5]. However, we can always choose a finite subset of any quadrangulation with periodic boundary conditions in such a way that the graph is still bipartite. This observation is crucial, as it is wellknown $[10,16,52]$ that WSK is ergodic at zero temperature for any bipartite graph and any number of states $q \geq 2$. Therefore, we can use WSK safely even at zero temperature for all the quadrangulations depicted in figures 2-4. (However, the ergodicity of WSK at $T=0$ cannot be taken for granted for non-bipartite lattices and certain values of the number of states $q$ : e.g., the triangular lattice for $q=4$ [53], or the kagome lattice for $q=3$ [54].)

As mentioned in section 2.2, it is clear from figures 2-4 that all the quadrangulations considered in this paper can be regarded as a Bravais lattice $\mathcal{B}_{\Gamma}$ with a basis formed by $n_{\Gamma}$ vertices.

Dealing with a Bravais lattice with a non-trivial basis is not hard, but it involves some extra work. To our knowledge, in the literature one can find only simple cases $[11,64]$, like the hexagonal lattice. Therefore, we will explain how to deal with a generic bipartite quadrangulation $\Gamma=\mathcal{Q}\left(G_{0}\right)=\left(V_{\Gamma}, E_{\Gamma}\right)$ associated to a dual pair 
$\left(G_{0}, G_{1}\right)$. Let us assume that the sublattice $G_{0}$ (resp. $\left.G_{1}\right)$ is the even (resp. odd) sublattice with vertex set $V_{0}$ (resp. $\left.V_{1}\right)$. Indeed, $V_{\Gamma}=V_{0} \cup V_{1}$.

In order to measure some observables (see below), we need to embed the graph $\Gamma$ in a torus, and assign to each vertex $x \in V_{\Gamma}$ a vector $\boldsymbol{x} \in \mathbb{R}^{2}$. Then, each vertex $x \in V$ is associated to a vector that can be written as:

$$
\boldsymbol{x}=\sum_{j=1}^{2} x_{j}^{\prime} \boldsymbol{\eta}_{j}+\sum_{j=1}^{n_{\Gamma}-1} \epsilon_{j} \boldsymbol{\mu}_{j}=\boldsymbol{x}^{\prime}+\sum_{j=1}^{n_{\Gamma}-1} \epsilon_{j} \boldsymbol{\mu}_{j}, \quad x_{1}^{\prime}, x_{2}^{\prime}=1, \ldots, L,
$$

where the unit vectors $\boldsymbol{\eta}_{1}, \boldsymbol{\eta}_{2} \in \mathbb{R}^{2}$ span the Bravais lattice $\mathcal{B}_{\Gamma}$, which has dimensions $L \times L$ (unit cells) with periodic boundary conditions. Then $\Gamma$ has $\left|V_{\Gamma}\right|=\left|V_{0}\right|+\left|V_{1}\right|=$ $n_{\Gamma} L^{2}$ vertices. The non-trivial content of the basis is given in (3.1) by the $n_{\Gamma}-1$ vectors $\left\{\boldsymbol{\mu}_{j}\right\}$. If we assume that one of the vertices of the basis (i.e., $x^{\prime}$ with vector $\boldsymbol{x}^{\prime}$ ) belongs to the Bravais lattice $\mathcal{B}_{\Gamma}$, then the vectors $\boldsymbol{\mu}_{j}$ give the position of the nontrivial vertices of the basis with respect to $\boldsymbol{x}^{\prime}$. Finally, the $\epsilon_{j}$ are the components of an $\left(n_{\Gamma}-1\right)$-dimensional vector $\boldsymbol{\epsilon}=\left(\epsilon_{1}, \epsilon_{2}, \ldots, \epsilon_{n_{\Gamma}-1}\right)$, that (according to the preceding discussion) can take only $n_{\Gamma}$ values: it can be either the zero vector $\boldsymbol{\epsilon}=\mathbf{0}$ (when the vertex $x$ belongs to $\mathcal{B}_{\Gamma}$ ), or any of the unit vectors of the standard basis of $\mathbb{R}^{n_{\Gamma}-1}$ : i.e., $\boldsymbol{\epsilon}=(0, \ldots, 0,1,0, \ldots, 0)$, when $x$ is a non-trivial vertex of the basis.

We define the sublattice magnetisations $\mathcal{M}_{i}$ (for $\left.i=0,1\right)$ as:

$$
\mathcal{M}_{i}=\sum_{x \in V_{i}} \boldsymbol{\sigma}_{x}=\sum_{x \in V_{i}} \sum_{\alpha=1}^{q} \boldsymbol{e}^{(\alpha)} \delta_{\sigma_{x}, \alpha}
$$

where $\boldsymbol{\sigma}_{x} \in \mathbb{R}^{q-1}$, and the unit vectors $\boldsymbol{e}^{(\alpha)}$ satisfy

$$
\boldsymbol{e}^{(\alpha)} \cdot \boldsymbol{e}^{(\beta)}=\frac{q \delta_{\alpha, \beta}-1}{q-1}
$$

As explained in section 2.3, we should compute both the staggered and the uniform susceptibilities. As the graph $\Gamma$ is bipartite, the natural staggering should be the one that assigns a +1 (resp. -1 ) to all vertices in $V_{0}$ (resp. $V_{1}$ ). This is obviously motivated by the Ising case. In our case, the staggered $\mathcal{M}_{\text {stagg }}$ an uniform $\mathcal{M}_{\mathrm{u}}$ magnetisations are given by

$$
\begin{aligned}
\mathcal{M}_{\text {stagg }} & =\mathcal{M}_{0}-\mathcal{M}_{1} \\
\mathcal{M}_{\mathrm{u}} & =\mathcal{M}_{0}+\mathcal{M}_{1}
\end{aligned}
$$

Then, the formulas for $\mathcal{M}_{\text {stagg }}^{2}$ and $\mathcal{M}_{\mathrm{u}}^{2}$ follow from $(3.2) /(3.3)$ :

$$
\begin{aligned}
\mathcal{M}_{\text {stagg }}^{2} & =\frac{q}{q-1} \sum_{\alpha=1}^{q}\left[\sum_{x \in V_{0}} \delta_{\sigma_{x}, \alpha}-\sum_{x \in V_{1}} \delta_{\sigma_{x}, \alpha}\right]^{2}-\frac{\left(\left|V_{0}\right|-\left|V_{1}\right|\right)^{2}}{q-1} \\
\mathcal{M}_{\mathrm{u}}^{2} & =\frac{q}{q-1} \sum_{\alpha=1}^{q}\left(\sum_{x \in V} \delta_{\sigma_{x}, \alpha}\right)^{2}-\frac{\left|V_{\Gamma}\right|^{2}}{q-1}
\end{aligned}
$$


The corresponding susceptibilities are defined as follows:

$$
\chi_{\text {stagg }}=\frac{1}{\left|V_{\Gamma}\right|}\left\langle\mathcal{M}_{\text {stagg }}^{2}\right\rangle, \quad \chi_{\mathrm{u}}=\frac{1}{\left|V_{\Gamma}\right|}\left\langle\mathcal{M}_{\mathrm{u}}^{2}\right\rangle
$$

and the corresponding dimensionless Binder-like ratios are given by

$$
R_{\text {stagg }}=\frac{\left\langle\left(\mathcal{M}_{\text {stagg }}^{2}\right)^{2}\right\rangle}{\left\langle\mathcal{M}_{\text {stagg }}^{2}\right\rangle^{2}}, \quad R_{\mathrm{u}}=\frac{\left\langle\left(\mathcal{M}_{\mathrm{u}}^{2}\right)^{2}\right\rangle}{\left\langle\mathcal{M}_{\mathrm{u}}^{2}\right\rangle^{2}} .
$$

The number of MC steps we have run for each simulation [i.e. for each triplet $(\Gamma, q, v)]$ is in the range $6 \times 10^{6}-10^{8}$, although most of them consist in $\approx 10^{7} \mathrm{MC}$ steps. In all cases, we have discarded between $10 \%$ and $20 \%$ of the total number of $\mathrm{MC}$ steps to allow the system to attain thermodynamic equilibrium. The error bars in our MC estimates were computed using the jackknife method (see e.g., [47, Section 5.7.5, and references therein]). With our statistics, we have been able to obtain error bars of relative size $\lesssim 0.1 \%$.

The number of MC steps discarded at the beginning of the simulations is also large enough for the system to reach its thermodynamic equilibrium. We have measured the integrated autocorrelation times $\tau_{\text {int }}$ for four quadrangulations of non-self-dual type: $\mathcal{Q}$ (diced), $\mathcal{Q}$ (martini), $\mathcal{Q}$ (ruby), and $G_{3}^{\prime \prime}$. This autocorrelation time is roughly speaking the number of MC steps between two statistically independent spin configurations [73], once the Markov-chain MC has reached equilibrium. In all MC simulations performed on these lattices, the worst case corresponds to the $\mathcal{Q}$ (martini) lattice with linear size $L=512$, giving $\tau_{\text {int }} \lesssim 90$. This means that for an average simulation of $10^{7} \mathrm{MC}$ steps, the number of discarded steps is at least $\gtrsim 1.1 \times 10^{4} \tau_{\text {int }}$ for all cases. This is more than enough to get rid of any initialisation bias.

For quadrangulations of self-dual type, the situation is even better: we have found that the WSK algorithm at the zero-temperature critical point does not suffer from CSD. This means that $\tau_{\text {int }}$ is (for any of these latices) uniformly bounded in the lattice linear size $L$. In our case, we have found that $\tau_{\text {int }} \lesssim 8$ for the four lattices considered in section 3.1.1. This result also agrees with the previous computation for the square-lattice 3 -state AF Potts model: $\tau_{\text {int }} \lesssim 8$ uniformly in $L$ [67].

\subsubsection{Quadrangulations of self-dual type}

In this section we will report the numerical results for the four quadrangulations of self-dual type that we have simulated: $\mathcal{Q}$ (hextri), $\mathcal{Q}$ (house), $\mathcal{Q}$ (martini-B), and $\mathcal{Q}(\mathrm{cmm}-\mathrm{pmm})$ (see figure 2).

For each lattice, we have first computed the Binder ratios (3.7), and found that all the curves $R_{\mathrm{a}}(v ; L)$ for a $\in\{$ stagg, $\mathrm{u}\}$, nicely overlap at $v=-1$, confirming part (1) of conjecture 1.1: the AF 3-state Potts model on a quadrangulation of self-dual type is critical at zero temperature $v_{c}=-1$. As in this case the critical temperature is known, we have fitted the zero-temperature susceptibilities to the standard power-law Ansatz

$$
\chi_{\mathrm{a}}(-1 ; L)=L^{(\gamma / \nu)_{\mathrm{a}}}\left[A+B L^{-\omega}+\cdots\right], \quad \mathrm{a} \in\{\operatorname{stagg}, \mathrm{u}\} .
$$


As a precaution against subdominant finite-size-scaling (FSS) corrections, we have systematically varied the minimum value $L_{\min }$ of the data included in the fit. In this way we obtain the results displayed in table 1.

If we plot the scaled susceptibilities $\chi_{\mathrm{a}} L^{-(\gamma / \nu)_{\mathrm{a}}}$ with $\mathrm{a} \in\{$ stagg, $\mathrm{u}\}$ for the $\mathcal{Q}$ (house) lattice, we obtain the plots displayed in figure 6 . It is clear that the curves for different values of the linear size $L$ overlap at the critical point $v_{c}=-1$, as expected. As the corresponding figures for the other three lattices are very similar to those shown in figure 6 , we refrain from showing them. (Notice that the plot of the scaled staggered susceptibility for the lattice $\mathcal{Q}$ (hextri) has already appeared in [51, figure 3].)

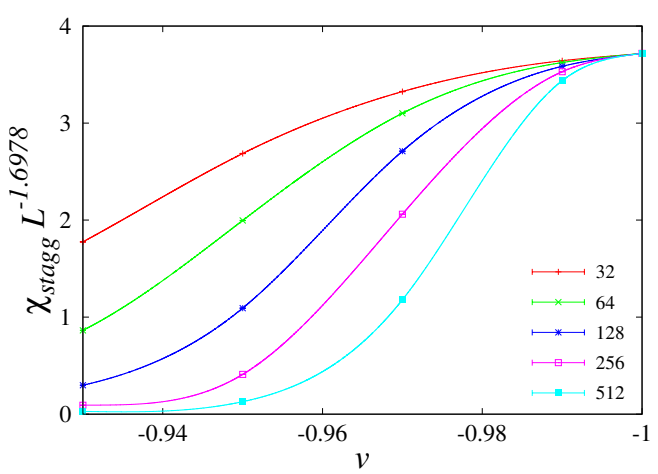

(a)

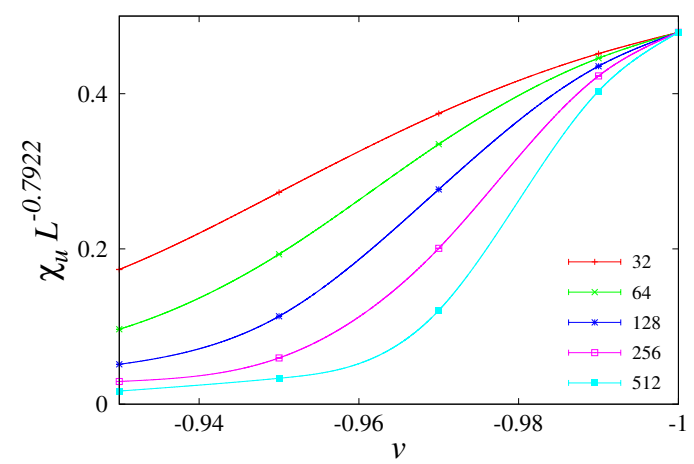

(b)

Figure 6: Scaled staggered $\chi_{\text {stagg }}(\mathrm{a})$ and uniform $\chi_{\mathrm{u}}(\mathrm{b})$ susceptibilities (see (3.6)) as a function of the temperature-like parameter $v$ for a $\mathcal{Q}$ (house) lattice of dimensions $L \times L$ and periodic boundary conditions. We show the data points for $L=32$ (red), $L=64$ (green), $L=128$ (navy blue), $L=256$ (pink), and $L=512$ (cyan). For each value of $L \leq 256$, we have also drawn a spline-interpolation curve joining the corresponding points to guide the eye. The error bars of the points are smaller than the corresponding symbols.

As both critical exponents $(\gamma / \nu)_{\text {stagg }}$ and $(\gamma / \nu)_{\mathrm{u}}$ depend on a single parameter, i.e., the stiffness $K$ (see $(2.14) /(2.15))$, we can estimate $K$ by using our numerical estimates for the former:

$$
\left.\frac{\gamma}{\nu}\right|_{\mathrm{stagg}}=2-\frac{\pi}{18 K},\left.\quad \frac{\gamma}{\nu}\right|_{\mathrm{u}}=2-\frac{2 \pi}{9 K} .
$$

The results of such fits are displayed on the fourth column of table 1. Indeed, all of them are smaller than the critical stiffness $K_{c}=\pi / 2 \approx 1.5708$.

As explained in section 2.3, we expect that the stiffness $K$ will depend on the lattice structure, and this feature is observed in table 1 . It is worth noticing that a potential counterexample to conjecture 1.1(a) will be provided by a quadrangulation of self-dual type $\Gamma$ for which $K>K_{c}=\pi / 2$. From table 1 , we see that the estimated values for $K$ do not vary much from that of the square lattice $K=\pi / 6$, and all of them 


\begin{tabular}{lcccc}
\hline \hline \multicolumn{1}{c}{$\Gamma$} & $(\gamma / \nu)_{\text {stagg }}$ & $(\gamma / \nu)_{\mathrm{u}}$ & $K$ & $\Delta$ \\
\hline $\mathcal{Q}(\mathrm{cmm}-\mathrm{pmm})$ & $1.71762(9)$ & $0.8691(5)$ & $0.6177(6)$ & 8 \\
$\mathcal{Q}$ (hextri) & $1.7024(3)$ & $0.8096(9)$ & $0.5865(6)$ & 6 \\
$\mathcal{Q}$ (house) & $1.6978(3)$ & $0.7922(4)$ & $0.5778(8)$ & 5 \\
$\mathcal{Q}$ (martini-B) & $1.6882(3)$ & $0.7557(9)$ & $0.5609(6)$ & 5 \\
square & $5 / 3 \approx 1.66667$ & $2 / 3 \approx 0.66667$ & $\pi / 6 \approx 0.52360$ & 4 \\
\hline \hline
\end{tabular}

Table 1: Critical exponents $(\gamma / \nu)_{\text {stagg }}$ and $(\gamma / \nu)_{\mathrm{u}}$, and the estimated value of the stiffness $K$ for the zero-temperature AF 3 -state Potts model on the quadrangulations of self-dual type $\Gamma$ studied in this paper. We also show the maximum degree $\Delta$ for each of these lattices. These results have already appeared in [51, table I]. We include for comparison the exact values for the square lattice.

are roughly speaking 2.5 times smaller than the critical value $K_{c}=\pi / 2 \approx 1.570796$. A closer inspection of table 1 reveals that the stiffness $K$ seems to grow weakly with the maximum degree of the lattice $\Delta$. Therefore, for all plane quadrangulations of self-dual type with maximum degree $\Delta \lesssim 8$, we expect that $K<K_{c}$. On the other hand, potential counterexamples to conjecture 1.1(a) might be found when $\Delta$ becomes very large, if these quadrangulations do exist at all. As a matter of fact, we chose the rather complicated quadrangulation $\mathcal{Q}(\mathrm{cmm}-\mathrm{pmm})$ with $\Delta=8$ to test this weak dependency of the stiffness $K$ on $\Delta$.

The dynamic behaviour of the WSK algorithm at the zero-temperature critical point found for these four systems is worth discussing in detail. Once a Markovchain $\mathrm{MC}$ has reached its equilibrium state, then, for any local observable $\mathcal{O}$, one can compute its integrated autocorrelation time $\tau_{\text {int }, \mathcal{O}}$ [73]. The integrated autocorrelation time $\tau_{\text {int }}$ is just the maximum over all observables of $\tau_{\text {int }, \mathcal{O}}$. Therefore, each $\tau_{\text {int }, \mathcal{O}}$ is a lower bound for the true value $\tau_{\text {int }}$. We have measured the autocorrelation times for the critical observables $\mathcal{M}_{\text {stagg }}^{2}$ and $\mathcal{M}_{\text {stagg }}^{2}$ (see table 2).

It is clear from this table that in all cases, $\tau_{\text {int }, \mathcal{M}_{\text {stagg }}^{2}} \lesssim \tau_{\text {int, } \mathcal{M}_{\mathrm{u}}^{2}} \lesssim 8$ uniformly in $L$, at least for the range of sizes considered in this paper $32 \leq L \leq 512$. For each lattice shown in table 2 , we see that both autocorrelation times $\tau_{\text {int, } \mathcal{M}_{\text {stagg }}^{2}}$ and $\tau_{\text {int }, \mathcal{M}_{\mathrm{u}}^{2}}$ are roughly independent of $L$ within errors. This observation gives a strong support to our conjecture that these integrated autocorrelation times are uniformly bounded in $L$; i.e., the absence of CSD for the WSK algorithm on this class of AF models. For the square-lattice case [67], another critical observable (the staggered polarisation) was considered, whose integrated autocorrelation time was also uniformly bounded in $L$, but this bound was larger than the corresponding magnetisation-square bounds. This example illustrates that we cannot rule out the possibility that there is another observable whose integrated autocorrelation time is larger than those quoted in table 2; but we expect that this autocorrelation time will be also uniformly bounded in $L$. If this is true, then the conclusions discussed above still hold, although the uniform bound on $\tau_{\text {int }}$ will be larger.

Finally, let us stress the empirical observation that the bounds displayed in table 2 


\begin{tabular}{r|cc|cc|cc|cc}
\hline \hline$\Gamma$ & \multicolumn{2}{|c|}{$\mathcal{Q}$ (martini-B) } & \multicolumn{2}{|c|}{$\mathcal{Q}$ (house) } & \multicolumn{2}{c|}{$\mathcal{Q}$ (hextri) } & \multicolumn{2}{c}{$\mathcal{Q}$ (cmm-pmm) } \\
$\Delta$ & \multicolumn{2}{|c|}{5} & \multicolumn{2}{|c|}{5} & \multicolumn{2}{c}{6} & \multicolumn{2}{c}{8} \\
\hline$L$ & $\mathcal{M}_{\text {stagg }}^{2}$ & $\mathcal{M}_{\mathrm{u}}^{2}$ & $\mathcal{M}_{\text {stagg }}^{2}$ & $\mathcal{M}_{\mathrm{u}}^{2}$ & $\mathcal{M}_{\text {stagg }}^{2}$ & $\mathcal{M}_{\mathrm{u}}^{2}$ & $\mathcal{M}_{\text {stagg }}^{2}$ & $\mathcal{M}_{\mathrm{u}}^{2}$ \\
\hline 32 & & & & & & & $6.2(1)$ & $7.2(1)$ \\
64 & $5.0(1)$ & $4.9(1)$ & $5.0(1)$ & $5.3(1)$ & $5.6(1)$ & $6.3(1)$ & $6.3(1)$ & $7.6(1)$ \\
128 & $5.0(1)$ & $5.0(1)$ & $5.1(1)$ & $5.4(1)$ & $5.7(1)$ & $6.5(1)$ & $6.5(1)$ & $8.0(1)$ \\
256 & $5.0(1)$ & $4.9(1)$ & $5.1(1)$ & $5.4(1)$ & $5.7(1)$ & $6.5(1)$ & $6.3(2)$ & $7.9(2)$ \\
384 & & & & & & & $6.2(2)$ & $7.7(3)$ \\
512 & $4.9(1)$ & $5.0(2)$ & $5.0(1)$ & $5.3(2)$ & $5.6(1)$ & $6.5(1)$ & & \\
\hline \hline
\end{tabular}

Table 2: Integrated autocorrelation times of the WSK algorithm for the the zerotemperature critical point of the AF 3-state Potts model on the quadrangulations $\Gamma$ of self-dual type considered in this work. For each lattice $\Gamma$, we show in the second row its maximum degree $\Delta$. We also display the integrated autocorrelation times $\tau_{\text {int, } \mathcal{O}}$ for the critical observables $\mathcal{O}=\mathcal{M}_{\text {stagg }}^{2}, \mathcal{M}_{\mathrm{u}}^{2}$ as a function of the linear size $L$ (in unit cells). The missing entries were not measured. The data for the square lattice can be found in [67].

for $\tau_{\text {int, } \mathcal{M}_{\mathrm{u}}^{2}}$ seem to (weakly) grow with the maximum degree $\Delta$ of the quadrangulation. Notice that for the square lattice with $\Delta=4$, the bound is $\tau_{\text {int, } \mathcal{M}_{\mathrm{u}}^{2}} \lesssim 5[67]$.

\subsubsection{Quadrangulations of non-self-dual type}

In this section we will report the numerical results for the seven quadrangulations of non-self-dual type that we have simulated (see figures 3-4). In four cases (namely, $\mathcal{Q}$ (diced), $\mathcal{Q}$ (martini-B), $\mathcal{Q}$ (ruby), and $G_{3}^{\prime \prime}$ ), we have measured both the static and dynamic observables. For the other three cases (i.e., $\mathcal{Q}$ (cross), $\mathcal{Q}$ (asanoha), and $G_{2}^{\prime \prime}$ ), we have focused on the static observables. Concerning the static observables, we have found that the staggered and uniform observables behave in the same way. Therefore, we will consider the former, and omit details about the latter. Moreover, as the plots for all lattices are quite similar, we will refrain from showing all the plots, and display only those for $\mathcal{Q}$ (ruby) in figure 7. (The plot of the scaled staggered susceptibility for the lattice $\mathcal{Q}$ (diced) has already appeared in [51, figure 4].)

First, we have computed the Binder ratio $R_{\text {stagg }}$ (see (3.7)). In all cases, we find that the curves $R_{\text {stagg }}(v ; L)$ cross at a non-zero value of the temperature. This common crossing point determines the position of the critical value $-1<v_{c}<0$ for each lattice. This critical value $v_{c}$, as well as the critical exponent $1 / \nu$, and the FSS correction exponent $\omega$ can be estimated by using Ansätze of the form

$$
R_{\text {stagg }}(v ; L)=R_{\text {stagg }}^{*}+a_{1}\left(v-v_{c}\right) L^{1 / \nu}+a_{2}\left(v-v_{c}\right)^{2} L^{2 / \nu}+b L^{-\omega}+\cdots,
$$

by omitting various subsets of terms, and varying the smallest value $L_{\min }$ of the data included in the fit. In this way we obtained stable fits for the parameters and their error bars. In all cases, the estimates for $1 / \nu$ agree well within statistical errors with 


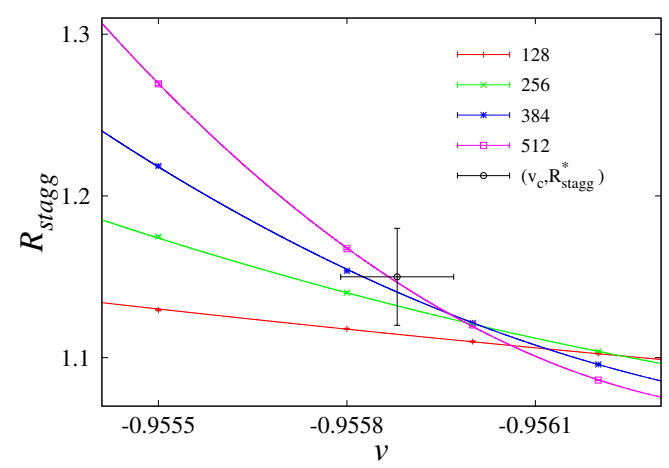

(a)

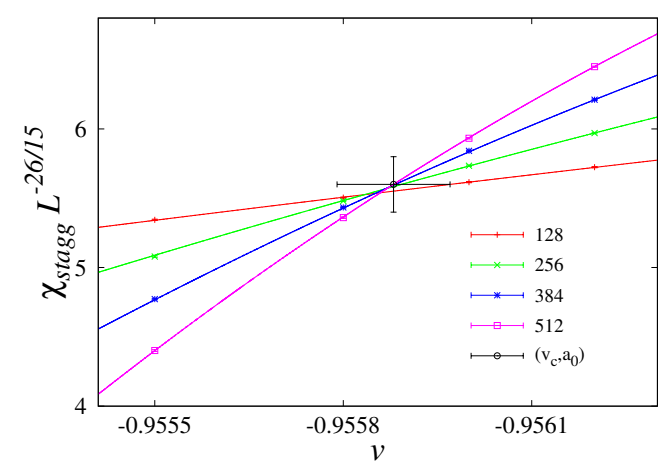

(b)

Figure 7: Binder cumulant $R_{\text {stagg }}$ (a) and scaled staggered susceptibility $L^{-26 / 15} \chi_{\text {stagg }}$ (b) as a function of the temperature-like parameter $v$ for a $\mathcal{Q}$ (ruby) lattice of dimensions $L \times L$ (unit cells) and periodic boundary conditions. We depict the data points for $L=128$ (red), $L=256$ (green), $L=384$ (navy blue), and $L=512$ (pink). The error bars of the points are smaller than the corresponding symbols. The curves correspond to our preferred fit $(3.10) /(3.11)$ with both $1 / \nu$ and $\gamma / \nu$ fixed to the exact values for the 3 -state Potts ferromagnet.

the expected value. We then redid the fits, with $1 / \nu$ fixed to its expected value; in this way we obtained better estimates for $v_{c}$. In figure $7(\mathrm{a})$ we show the Binder cumulant $R_{\text {stagg }}(v ; L)$ for the quadrangulation $\mathcal{Q}$ (ruby). We display our measurements for values of $v$ close enough to the critical value $v_{c}$, and $L=32,64,128,256,512$. Our preferred estimate for the point $\left(v_{c}, R_{\text {stagg }}^{*}\right)$ is also shown.

In the same way, we fitted the staggered susceptibility $\chi_{\text {stagg }}($ see $(3.6))$. In this case, the form of our Ansätze should be

$$
\begin{aligned}
& \chi_{\text {stagg }}(v ; L)=L^{(\gamma / \nu)_{\operatorname{stagg}}}\left[a_{0}+a_{1}\left(v-v_{c}\right) L^{1 / \nu}+a_{2}\left(v_{c}-v\right)^{2} L^{2 / \nu}\right. \\
& \left.+b L^{-\omega}+\cdots\right] \text {. }
\end{aligned}
$$

Again, we checked that the estimates for both $1 / \nu$ and $\gamma / \nu$ agree well with the expected values within statistical errors. Then, we redid the fits with (some of) these parameters fixed to their expected values to obtain more precise estimates for $v_{c}$. In figure $7(\mathrm{a})$ we show the scaled staggered susceptibility $\chi_{\text {stagg }}(v ; L) L^{-26 / 15}$ for the quadrangulation $\mathcal{Q}$ (ruby). We display our numerical data for values of $v$ sufficiently close to the critical value $v_{c}$, and $L=32,64,128,256,512$. Our preferred estimate for the point $\left(v_{c}, \chi_{\text {stagg }}^{*} L^{-26 / 15}\right)$ is also shown. Note that the critical exponent $(\gamma / \nu)_{\text {stagg }}=26 / 15$ is the one expected for the FM 3-state Potts model, and it is not the one estimated using (3.11).

The estimates of $1 / \nu$ and $v_{c}$ obtained from $(3.10) /(3.11)$ are combined, and the final result is displayed in table 3 . In the same table we will show the value of $R_{\text {stagg }}^{*}$ 
and $(\gamma / \nu)_{\text {stagg }}$ obtained in (3.10) and (3.11), respectively. The final error bars are obtained by combining the statistical errors and the systematic errors. These ones are computed by comparing different estimates coming from all good fits obtained in the above described procedure.

\begin{tabular}{llrrl}
\hline \hline \multicolumn{1}{c}{$\Gamma$} & \multicolumn{1}{c}{$v_{c}$} & $(\gamma / \nu)_{\text {stagg }}$ & $\nu$ & \multicolumn{1}{c}{$R_{\text {stagg }}^{*}$} \\
\hline $\mathcal{Q}$ (ruby) & $-0.95588(9)$ & $1.737(5)$ & $0.80(5)$ & $1.15(3)$ \\
$\mathcal{Q}$ (diced) & $-0.94075(12)$ & $1.737(6)$ & $0.83(7)$ & $1.17(2)$ \\
diced & $-0.860599(4)$ & $1.737(4)$ & $0.81(2)$ & $1.170(7)$ \\
$G_{2}^{\prime \prime}$ & $-0.82278(4)$ & $1.737(5)$ & $0.82(2)$ & $1.17(1)$ \\
$\mathcal{Q}$ (cross) & $-0.80057(4)$ & $1.736(7)$ & $0.85(3)$ & $1.19(2)$ \\
$\mathcal{Q}$ (martini) & $-0.77454(6)$ & $1.735(4)$ & $0.83(3)$ & $1.16(1)$ \\
$G_{3}^{\prime \prime}$ & $-0.72278(2)$ & $1.736(4)$ & $0.82(2)$ & $1.17(1)$ \\
$\mathcal{Q}$ (asanoha) & $-0.72033(3)$ & $1.735(4)$ & $0.83(2)$ & $1.16(2)$ \\
\hline Prediction & & $26 / 15 \approx 1.73333$ & $5 / 6 \approx 0.8333$ & $1.1711(5)$ \\
\hline \hline
\end{tabular}

Table 3: Critical temperature $v_{c}$, critical exponents $(\gamma / \nu)_{\text {stagg }}$ and $\nu$, and critical value of the Binder cumulant $R_{\text {stagg }}^{*}$ for the AF 3-state Potts model on quadrangulations of non-self-dual type $\Gamma$. We also include for comparison the results for the diced lattice [45], and the last line (labeled 'Prediction') shows the corresponding values for the FM 3-state Potts model $[18,66]$. The results for $\mathcal{Q}$ (ruby), $\mathcal{Q}$ (diced), $\mathcal{Q}$ (martini), and $G_{3}^{\prime \prime}$ have already appeared in [51, table III].

If we look at the estimates displayed in table 3, we find a rather good agreement with the prediction of conjecture 1.1(b). Moreover, as all MC simulations are statistically independent, we can test the hypothesis that the values for $(\gamma / \nu)_{\text {stagg }}, \nu$, and $R_{\mathrm{stagg}}^{*}$ are constant among this class of quadrangulations. A fit to a constant reveals that

$$
\begin{array}{rllll}
\left.\frac{\gamma}{\nu}\right|_{\text {stagg }}=1.736(2), & \chi^{2}=0.29, & \text { DF }=7, & \text { CL }=99.9 \% \\
\nu=0.823(9), & \chi^{2}=1.68, & \text { DF }=7, & \text { CL }=97.6 \% \\
R_{\text {stagg }}^{*}=1.168(4), & \chi^{2}=2.53, & \text { DF }=7, & \text { CL }=92.4 \%
\end{array}
$$

where DF is the number of degrees of freedom, and CL is the confidence level of the fit. The estimates agree within less than 1.5 standard deviations from those on the row labeled 'Prediction' in table 3. Therefore, we have found that for all the quadrangulations of non-self-dual type considered in this work, the phase transition separating the low- and high-temperature phases is of second order, and that this finite-temperature critical point belongs to the universality class of the FM 3-state Potts model (i.e., identical critical exponents and universal amplitudes). Therefore, all cases studied in this section are in full agreement with conjecture 1.1(b).

Finally, let us discuss in detail what happens for the special cases $\mathcal{Q}$ (diced) and $\mathcal{Q}$ (ruby). Please recall that for these two lattices, both sublattices $G_{0}$ and $G_{1}$ have 
the same number of vertices, and the general argument leading to conjecture 1.1(b) does not work in a straightforward sense. We are now interested in investigating the typical ordering in each sublattice. To achieve this goal, we will consider all possible sub-sublattices of each of four quadrangulations of non-self-dual type studied in this section (namely, $\mathcal{Q}$ (diced), $\mathcal{Q}$ (ruby), $\mathcal{Q}$ (martini), and $G_{3}^{\prime \prime}$ ), and measure their magnetisation. These sub-sublattices are depicted in figure 8.

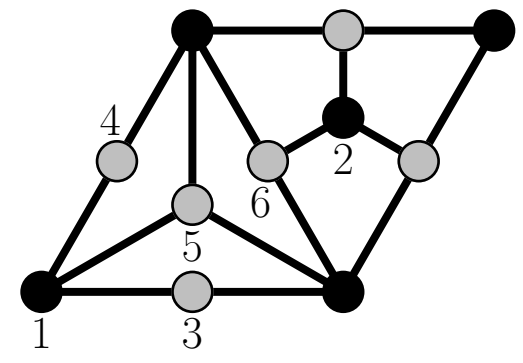

(a)

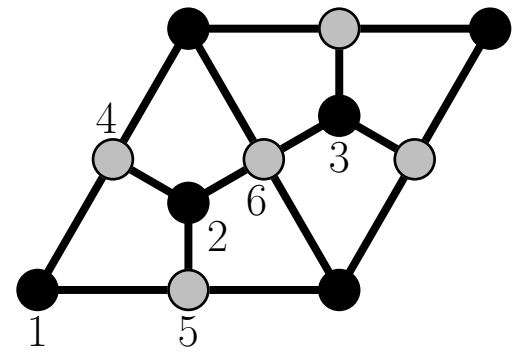

(c)

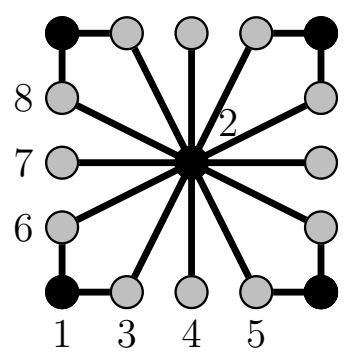

(b)

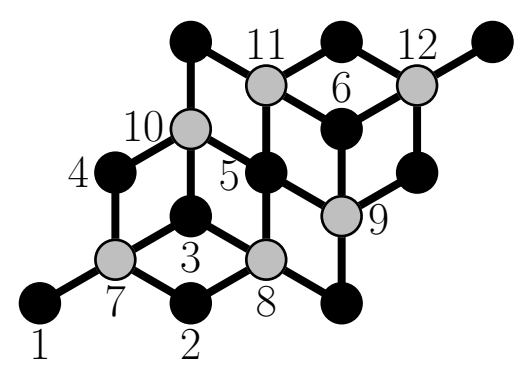

(d)

Figure 8: Unit cells for some of the quadrangulations $\Gamma$ of non-self-dual type depicted in figures 3 and 4: (a) $\mathcal{Q}$ (martini); (b) $G_{3}^{\prime \prime}$; (c) $\mathcal{Q}$ (diced); and (d) $\mathcal{Q}$ (ruby). Each quadrangulation $\Gamma$ is associated to a dual pair $\left(G_{0}, G_{1}\right)$. The black (resp. gray) vertices belong to $G_{0}$ (resp. $G_{1}$ ). In each unit cell, the numbers label the different sub-sublattices it contains.

The vertex set of each one of these four lattices $\Gamma$ can be partitioned into $n_{\Gamma}$ disjoint sets $V_{s}$ (see section 2.2). Then, for each sub-sublattice $s$, we can define the vector magnetisation

$$
\mathcal{M}^{(s)}=\sum_{\alpha=1}^{3} m_{\alpha}^{(s)} \boldsymbol{e}^{(\alpha)}
$$

where the unit vectors $\boldsymbol{e}^{(\alpha)}$ are given in (3.3), and

$$
m_{\alpha}^{(s)}=\frac{1}{\left|V_{s}\right|} \sum_{x \in V_{s}} \delta_{\sigma_{x}, \alpha}
$$

is the fraction of the spins in $V_{s}$ taking the value $\alpha \in\{1,2,3\}$. We then define the 
mean squared magnetisation density (MSMD) for each sub-sublattice $s$ as:

$$
M_{s}^{2}=\left\langle\mathcal{M}^{(s)} \cdot \mathcal{M}^{(s)}\right\rangle
$$

This quantity measures the FM order of the spins within each sub-sublattice $s$. If the spins in $s$ are completely FM ordered (i.e., they take a common value), then $M_{s}^{2}=1$; if the spins in $s$ take randomly two distinct values, then $M_{s}^{2}=1 / 4$; and if the spins in $s$ are completely uncorrelated, then $M_{s}^{2}=0$.

The unit cell of the lattice $\mathcal{Q}$ (martini) is shown in figure $8(\mathrm{a})$. The black (resp. gray) dots represent the vertices in $G_{0}$ (resp. $G_{1}=$ martini), and the labels refer to its six different sub-sublattices. The sublattice $G_{0}$ (resp. $G_{1}$ ) contains $\left|V_{0}\right|=2 L^{2}$ (resp. $\left|V_{1}\right|=4 L^{2}$ ) vertices, where $L$ is the linear size of the underlying triangular lattice. The sublattice with less vertices $G_{0}$ contains two sub-sublattices: one with degree-9 vertices (labeled 1 ), and another with degree-3 vertices (labeled 2 ). The corresponding MSMD are $M_{1}^{2} \approx 0.97$ and $M_{2}^{2} \approx 0.48$. $G_{1}$ can be decomposed into four sublattices (each of them with degree-3 vertices and labeled 3, 4, 5, and 6). For all of them, $M_{s}^{2} \approx 0.25$.

The unit cell of the lattice $G_{3}^{\prime \prime}$ is depicted in figure $8(\mathrm{~b})$. In this case $G_{0}=G_{2}^{\prime}$ contains $2 L^{2}$ vertices, while $G_{1}$ (= decorated $4-8$ lattice) has $6 L^{2}$ vertices, where $L$ is the linear size of the underlying square lattice. $G_{0}$ can be split into two subsublattices: one with degree- 12 vertices (labeled 2 ) and $M_{2}^{2} \approx 0.99$, and another with degree- 4 vertices (labeled 1 ) and $M_{1}^{2} \approx 0.69$. Moreover, $G_{1}$ can also be split into two degree-2 sublattices (labeled 4 and 7 ) and four degree-3 sublattices (labeled 3, 4, 6, and 8). For all of them, $M_{s}^{2} \approx 0.25$.

In both lattices, $\left|V_{0}\right|<\left|V_{1}\right|$, and the behavior of the MSMD for each sub-sublattice agrees qualitatively with our conclusions based on the height representation: the spins living on the sublattice with less vertices are FM ordered, while on the other sublattice, the spins take randomly the other two values. Notice however, that the ordering in $G_{0}$ is not uniform: the sub-sublattice with vertices of higher degree is almost completely ordered, while the other sub-sublattice is only partially ordered (i.e., $1 / 4<0.48 \lesssim M_{a}^{2} \lesssim 0.69<1$ ). On the other hand, all the sub-sublattices in $G_{1}$ take two random values $M_{a}^{2} \approx 1 / 4$. This difference with respect to the scenario presented in section 2.3 is probably due to fluctuations around the three ideal states (which were ignored in section 2.3).

Let us now consider the other two cases for which $\left|V_{0}\right|=\left|V_{1}\right|$. The unit cell of the lattice $\mathcal{Q}$ (diced) is shown in figure $8(\mathrm{c})$. In this case, both sublattices have $3 L^{2}$ vertices. The diced sublattice $G_{0}$ contains a degree- 6 sublattice (labeled 1 ), and two degree- 3 sublattices (labeled 2 and 3 ). The corresponding MSMD are $M_{1}^{2} \approx 0.49$ and $M_{2}^{2} \approx M_{3}^{2} \approx 0.28$, respectively. The kagome sublattice $G_{1}$ contains three degree- 4 sublattices (labeled 4 to 6 ) and for all of them, we obtain $M_{s}^{2} \approx 0.20$.

Finally, the unit cell of the lattice $\mathcal{Q}$ (ruby) is shown in figure $8(\mathrm{~d})$. In this case both sublattices have $6 L^{2}$ vertices. The $G_{0}=\mathcal{Q}$ (diced) sublattice contains a degree6 sub-sublattice (labeled 1), three degree-4 sub-sublattices (labeled 2, 4, and 5), and two degree-3 sub-sublattices (labeled 3 and 6 ). The corresponding MSMD are $M_{1}^{2} \approx 0.47, M_{2}^{2} \approx 0.32$, and $M_{3}^{2} \approx 0.22$ (all sub-sublattices with the same degree 
have approximately the same MSMD). The ruby sublattice $G_{1}$ has six degree- 4 subsublattices (labeled 7 to 12 ) with $M_{7}^{2} \approx 0.20$.

We observe that, when $\left|V_{0}\right|=\left|V_{1}\right|$, the same conclusions hold qualitatively; but there are significant quantitative changes. First of all, the most ordered sub-sublattice is that of $G_{0}$ with the largest degree (as in the generic case); but now it is only partially FM ordered: $1 / 4<0.48 \approx M_{a}^{2}<1$. Secondly, the other sub-sublattices are less ordered: $M_{a}^{2}$ is in the range $0.22-0.28$, which is roughly speaking similar to $1 / 4$. This means that the spins in those sub-sublattices basically take two values at random. Finally, all sub-sublattices of $G_{1}$ are less ordered than for the generic case: $M_{a}^{2} \approx 0.20<1 / 4$. Again these differences could be attributed to fluctuations around the three ideal states; but for this particular case $\left(\left|V_{0}\right|=\left|V_{1}\right|\right)$ these fluctuations look larger than for the generic one $\left(\left|V_{0}\right|<\left|V_{1}\right|\right)$. In conclusion, we see that, even for the most involved cases, the sublattice $G_{0}$ containing the sub-sublattice of largest degree is the one that is more ordered; while the other sublattice $G_{1}$ is disordered. These results give a stronger empirical support to conjecture 1.1(b).

\begin{tabular}{r|rr|rr|rr|rr}
\hline \hline$\Gamma$ & \multicolumn{2}{|c|}{$\mathcal{Q}$ (diced) } & \multicolumn{2}{c|}{$\mathcal{Q}$ (martini) } & \multicolumn{2}{c|}{$G_{3}^{\prime \prime}$} & \multicolumn{2}{c}{$\mathcal{Q}($ ruby $)$} \\
\hline$v$ & \multicolumn{2}{|c|}{-0.94071} & \multicolumn{2}{c}{-0.77454} & \multicolumn{2}{c}{-0.72278} & \multicolumn{2}{c}{-0.95588} \\
$L$ & $\mathcal{M}_{\text {stagg }}^{2}$ & $\mathcal{M}_{\mathrm{u}}^{2}$ & $\mathcal{M}_{\text {stagg }}^{2}$ & $\mathcal{M}_{\mathrm{u}}^{2}$ & $\mathcal{M}_{\text {stagg }}^{2}$ & $\mathcal{M}_{\mathrm{u}}^{2}$ & $\mathcal{M}_{\text {stagg }}^{2}$ & $\mathcal{M}_{\mathrm{u}}^{2}$ \\
\hline 16 & & & & & & & $9.3(1)$ & $10.2(1)$ \\
24 & & & & & $19(3)$ & $18.3(1)$ & & \\
32 & $11.7(1)$ & $13.3(1)$ & $21.1(1)$ & $15.4(1)$ & $27(7)$ & $22.0(2)$ & $11.6(1)$ & $13.6(1)$ \\
40 & & & & & $31(7)$ & $24.9(2)$ & & \\
48 & & & & & $28(14)$ & $27.6(3)$ & & \\
56 & & & & & $21(8)$ & $30.3(2)$ & & \\
64 & $14.6(1)$ & $16.7(1)$ & $30.1(2)$ & $27.0(2)$ & $49(33)$ & $32.3(4)$ & $14.1(1)$ & $16.4(1)$ \\
72 & & & & & $36(22)$ & $34.0(4)$ & & \\
128 & $18.8(1)$ & $20.9(2)$ & $42.7(4)$ & $40.3(4)$ & $57(29)$ & $46.1(5)$ & $17.6(3)$ & $19.9(3)$ \\
256 & $25.2(2)$ & $27.1(2)$ & $59.5(9)$ & $57.2(8)$ & & $65(1)$ & $22.8(4)$ & $24.8(4)$ \\
384 & & & & & & & $27.5(5)$ & $29.4(6)$ \\
512 & $33.3(9)$ & $35.9(7)$ & $88(3)$ & $79(3)$ & & $87(3)$ & $31(2)$ & $33(2)$ \\
\hline \hline
\end{tabular}

Table 4: Integrated autocorrelation times of the WSK algorithm for the the finitetemperature critical point of the AF 3-state Potts model on four of the quadrangulations $\Gamma$ of non-self-dual type considered in this work. For each lattice $\Gamma$, we show the integrated autocorrelation times $\tau_{\text {int, } \mathcal{O}}$ for the observables $\mathcal{O}=\mathcal{M}_{\text {stagg }}^{2}, \mathcal{M}_{\mathrm{u}}^{2}$ as a function of the linear size $L$ (in unit cells). The second row (labeled ' $v$ ') shows the temperature used to measure these estimates. The missing entries were not measured or could not be estimated (like the $L=256,512$ entries in the first column for $G_{3}^{\prime \prime}$ ).

Now let us take a look at the dynamic behaviour of the WSK algorithm for the AF 3 -state Potts model on the four quadrangulations considered above: $\mathcal{Q}$ (martini), $G_{3}^{\prime \prime}$, $\mathcal{Q}$ (diced) and $\mathcal{Q}$ (ruby). We have measured the integrated autocorrelation times $\tau_{\text {int }, \mathcal{O}}$ 
for the operators $\mathcal{O}=\mathcal{M}_{\text {stagg }}^{2}, \mathcal{M}_{\mathrm{u}}^{2}$. The raw data is displayed in table 4 . The second row of this table shows the temperature $v$ used in the MC simulations to estimate these autocorrelation times. Notice that this temperature is our preferred estimate for $v_{c}$, except for the lattice $\mathcal{Q}$ (diced). In this case $v=-0.94071$, which differs from $v_{c}=-0.94075(12)$ by just one third of one standard deviation for the latter value.

Table 4 shows that the autocorrelation time for $\mathcal{M}_{\text {stagg }}^{2}\left(\right.$ resp. $\mathcal{M}_{\mathrm{u}}^{2}$ ) is greater than the other one for $\mathcal{Q}$ (martini) and $G_{3}^{\prime \prime}$ (resp. $\mathcal{Q}$ (diced) and $\mathcal{Q}$ (ruby)). It seems that for those generic quadrangulations of non-self-dual type (with $\left|V_{0}\right|<\left|V_{1}\right|$ ) the slowest mode is $\mathcal{M}_{\text {stagg }}^{2}$, while for those quadrangulations with $\left|V_{0}\right|=\left|V_{1}\right|$, the slowest mode is $\mathcal{M}_{\mathrm{u}}^{2}$.

We have fitted the data of table 4 to a power-law Ansatz:

$$
\tau_{\text {int }, \mathcal{O}}=A_{\mathcal{O}}+B_{\mathcal{O}} L^{z_{\text {int }, \mathcal{O}}}
$$

where $z_{\text {int, } \mathcal{O}}$ is the corresponding dynamical critical exponent. Again, as a precaution against FSS artifacts, we have systematically varied the minimum value $L_{\min }$ of the data included in the fit. In some cases, a better and more stable fit is obtained by fixing $A_{\mathcal{O}}=0$. In this way we obtain the results displayed in table 5 . For completeness, we have also added data for the diced lattice. In this case, we have used (unpublished) dynamic data corresponding to [45], and the analysis was performed by using the FSS Ansatz (3.11) adapted to (3.16).

\begin{tabular}{cllrrrr}
\hline \hline$\Gamma$ & \multicolumn{1}{c}{$\mathcal{O}$} & \multicolumn{1}{c}{$z_{\text {int }, \mathcal{O}}$} & $L_{\min }$ & $\chi^{2}$ & DF & CL \\
\hline $\mathcal{Q}$ (diced) & $\mathcal{M}_{\text {stagg }}^{2}$ & $0.55(3)$ & 32 & 1.59 & 2 & $45 \%$ \\
& $\mathcal{M}_{\mathrm{u}}^{2}$ & $0.45(4)$ & 32 & 2.17 & 2 & $34 \%$ \\
\hline $\mathcal{Q}$ (martini) & $\mathcal{M}_{\text {stagg }}^{2}$ & $0.49(3)$ & 32 & 1.78 & 2 & $41 \%$ \\
& $\mathcal{M}_{\mathrm{u}}^{2}$ & $0.50(2)$ & $128^{\dagger}$ & 0.32 & 1 & $57 \%$ \\
\hline$G_{3}^{\prime \prime}$ & $\mathcal{M}_{\text {stagg }}^{2}$ & $1.0(6)$ & $48^{\dagger}$ & 0.88 & 3 & $83 \%$ \\
& $\mathcal{M}_{\mathrm{u}}^{2}$ & $0.48(2)$ & $128^{\dagger}$ & 1.21 & 1 & $27 \%$ \\
\hline $\mathcal{Q}$ (ruby) & $\mathcal{M}_{\text {stagg }}^{2}$ & $0.46(6)$ & $256^{\dagger}$ & 0.033 & 1 & $86 \%$ \\
& $\mathcal{M}_{\mathrm{u}}^{2}$ & $0.42(6)$ & $256^{\dagger}$ & 0.0054 & 1 & $94 \%$ \\
\hline diced [45] & $\mathcal{M}_{\text {stagg }}^{2}$ & $0.48(2)$ & 48 & 15.82 & 16 & $47 \%$ \\
& $\mathcal{M}_{\mathrm{u}}^{2}$ & $0.47(7)$ & 192 & 3.71 & 8 & $88 \%$ \\
\hline \hline
\end{tabular}

Table 5: Dynamic critical exponents $z_{\text {int, } \mathcal{M}_{\text {stagg }}^{2}}$ and $z_{\text {int, } \mathcal{M}_{\mathrm{u}}^{2}}$ for the WSK algorithm for the AF 3-state Potts model on quadrangulations of non-self-dual type $\Gamma$. The estimates for these critical exponents have been obtained using the Ansatz (3.16) using data with $\geq L_{\min }$. For each estimate, we show the corresponding values of $\chi^{2}$, the number of degrees of freedom (DF) and the confidence level (CL) of the fit. The symbol $^{\dagger}$ in the value of $L_{\text {min }}$ means that the fit has been done with $A_{\mathcal{O}}=0$. For the diced lattice, we have used (unpublished) dynamic data from [45], and an FSS Ansatz similar to $(3.11) /(3.16)$ for the corresponding autocorrelation times. 
A closer look at this table reveals that all dynamic critical exponents are roughly consistent within errors. A fit to a constant shows that this observation is true:

$$
\begin{aligned}
& z_{\text {int }, \mathcal{M}_{\text {stagg }}^{2}}=0.50(1), \quad \chi^{2}=4.98, \quad \mathrm{DF}=4, \quad \mathrm{CL}=47 \% \text {, } \\
& z_{\text {int }, \mathcal{M}_{\mathrm{u}}^{2}}=0.48(2), \quad \chi^{2}=3.02, \quad \mathrm{DF}=4, \quad \mathrm{CL}=56 \% \text {. }
\end{aligned}
$$

Finally, these two values are also consistent within 1-1.5 standard deviations. If these exponents are in fact equal, then our preferred estimate (taking into account the statistical non-independence of the two estimates) would be

$$
z_{\text {int }}=0.49(2)
$$

This value is close to the estimate of the dynamic critical exponent for the SW [75] algorithm for the square-lattice FM 3-state Potts model $z_{\text {int, } \mathcal{M}^{2}}=0.475(6)[18,66]$.

\subsection{Transfer matrices}

In this section we will show some results using the TM approach in the FK representation for the quadrangulations $\mathcal{Q}$ (hextri) and $\mathcal{Q}$ (house) of self-dual type. In particular, our goal is to show numerically that $(q, v)=(3,-1)$ is a critical point with central charge $c=1$, in agreement with conjecture 1.1(a). Therefore, we will focus on the chromatic polynomial case $v=-1$, and see how the free energy behaves as $q$ is varied in an interval around $q=3$. We will consider strip graphs of this lattice with cylindrical boundary conditions; i.e., periodic (resp. free) boundary conditions in the transverse (resp. longitudinal) direction. The TM for a strip graph of width $L$, and the chromatic polynomial $Z_{L \times N}(q,-1)$ for a strip graph of size $L \times N$ can be obtained in terms of join $\mathrm{J}_{i j}$ and detach $\mathrm{D}_{j}$ operators (acting on a suitable connectivity space), as it is shown in detail, e.g., in [31,32,68].

We have chosen cylindrical boundary conditions because they are easier to deal with, so they allow us to study strip graphs with larger widths $L \leq 14$; but the limiting curves $\mathcal{B}_{L}$ (in the complex $q$-axis) along which the chromatic zeros accumulate in the limit $N \rightarrow \infty$, may or may not cross the real $q$ axis. For instance, for $L=$ 4,12 , the finite-size estimates $q_{c}(L)$ for the parameter $q_{c}(\mathcal{Q}$ (hextri)) do not exist. (Toroidal boundary conditions [33] are expected to provide estimates $q_{c}(L)$ for any $L$ large enough, and these estimates are also expected to converge faster to their thermodynamic limit $q_{c}$; but are technically more difficult to handle and the range of amenable widths is therefore smaller: in particular $L \leq 8$ for $\mathcal{Q}$ (hextri).)

\subsubsection{Transfer matrix for $\mathcal{Q}$ (hextri)}

Let us start with the TM description of the $\mathcal{Q}$ (hextri) lattice (see figure 9 ). Notice that for this lattice, the width $L$ should be an even integer, and there are two different classes of vertices (depicted as white and gray dots in figure 9). The building of the chromatic-polynomial TM for this strip graph with cylindrical boundary conditions is shown in figure $9(\mathrm{a})$. This matrix can be written simply as

$$
\mathrm{T}=\mathrm{V} \cdot \mathrm{H} \cdot \mathrm{V}_{0}^{(\text {even })} \cdot \mathrm{H}
$$


where the operators act from right to left. The usual 'horizontal' H and 'vertical' V operators can be decomposed as products of sparse matrices

$$
\mathrm{H}=\left(\prod_{i=1}^{L-1} \mathrm{Q}_{i, i+1}\right) \cdot \mathrm{Q}_{L, 1}, \quad \mathrm{~V}=\prod_{i=1}^{L} \mathrm{P}_{i}
$$

which in turn, are written in terms of the join/detach operators:

$$
\mathrm{Q}_{i j}=\mathbb{1}-\mathrm{J}_{i j}, \quad \mathrm{P}_{i}=-\mathbb{1}+\mathrm{D}_{i},
$$

where $\mathbb{1}$ is the identity operator. The operator $\mathrm{V}_{0}^{(\mathrm{even})}$ is just the product of detach operators on the sites labelled by an even number:

$$
\mathrm{V}_{0}^{(\text {even })}=\prod_{i=1}^{L / 2} \mathrm{D}_{2 i}
$$

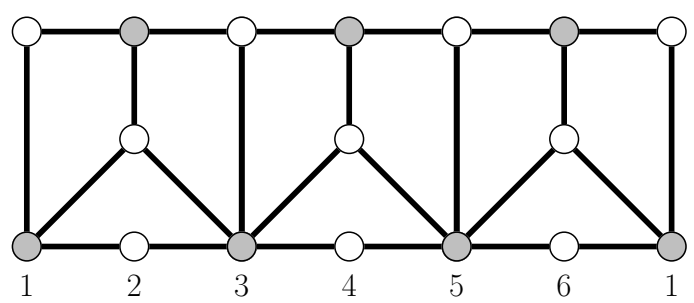

(a)

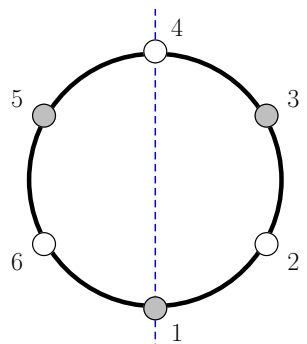

(b)

Figure 9: (a) TM construction for a layer of a strip graph of the $\mathcal{Q}$ (hextri) lattice of width $L=6$ and cylindrical boundary conditions. There are two classes of nonequivalent vertices, depicted respectively, as white and gray dots. (b) Another representation of the bottom row of panel (a) so that the periodic boundary conditions in the transverse direction are evident. The strip graph is symmetric with respect to the vertical dashed line going through vertices 1 and $1+L / 2=4$.

These operators act on the space of non-crossing connectivities for $L$ points on a circle. Its dimension is given by the Catalan number $C_{L}[68$, table 2]. We can reduce this dimension by taking into account that we are dealing with the chromatic polynomial, so we only need non-crossing non-nearest-neighbour connectivities, whose number is given by the Riordan numbers $R_{L}$ for $L \geq 2[68$, table 2$]$. We can reduce this dimension by taking also into account the symmetries of the $\mathcal{Q}$ (hextri) strip: i.e. rotations by two units (because of the non-equivalence of all points in the strip) and reflections by a line going through the vertices labelled 1 and $1+L / 2$ [see figure $9(\mathrm{~b})$ ]. Once all these symmetries are accounted for, the dimension of the corresponding connectivity space is given by $1,3,7,24,87,437,2379$, and 14843 for $L=2,4, \ldots, 16$. 
We have implemented this procedure by writing a PERL script, and we have been able to compute the TM for even widths $2 \leq L \leq 14$. We have checked the results by computing symbolically the chromatic polynomial for strip graphs of size $L \times N$ for $2 \leq N \leq N_{\max }$, and comparing them to the results provided by other well-tested symbolic programs $[6,21]$. The agreement is indeed perfect. For $L \leq 10, N_{\max }=20$; but for larger values of the width, the above-mentioned programs were unable to obtain results for some 'large' values of $N$ in a sensible amount of CPU time. In particular, for $L=12, N_{\max }=18$, and for $L=14, N_{\max }=4$.

Then, for each width $L$, we have computed the free energy in the interval $q \in[2,4]$ in terms of the dominant eigenvalue $\lambda_{*}$ of the corresponding TM:

$$
f_{L}(q,-1)=\frac{1}{L} \log \left|\lambda_{*}\right|
$$

We can extract the estimate for the central charge $c_{L}(q,-1)$ of a critical model by fitting the corresponding finite-size free energy (3.23) to the CFT Ansatz

$$
f_{L}(q,-1)=f_{\mathrm{bulk}}(q,-1)+\frac{G \pi c_{L}(q,-1)}{6 L^{2}}
$$

where $G=4 / \sqrt{3}$ is the geometrical factor for the $\mathcal{Q}$ (hextri) lattice when using the above construction.

Remark. Our definition of the free energy density (3.23) does not coincide with the usual definition of the free energy density per vertex by a factor of 2 . This implies that the geometric factor $G$ is different for the one obtained in this latter case. See appendix A for the computation of $G$.

Figure 9(b) shows clearly that the properties of the strip under reflections depend on whether $L \equiv 0,2(\bmod 4)$. In the former (resp. latter) case, the reflection axis goes trough two vertices belonging to distinct (resp. the same) sublattices. Therefore, we expect parity effects mod 4 in the free energy $f_{L}(q,-1)$. Again, as a precaution against subdominant FSS corrections, we have systematically varied the minimum value $L_{\min }$ of the data included in the fit. Therefore, we have used in each fit to the Ansatz (3.24) the values $L=L_{\min }, L_{\min }+4$. For each value of $L=L_{\min }$, we obtained a curve $c_{L}(q,-1)$ that displays a maximum at the point $\left(q_{\max }(L), c_{\max }(L)\right)$, where $c_{\max }(L)=c\left(q_{\max }(L),-1\right)$. These curves are depicted in figure 10(a), and the corresponding values for their maxima are shown table 6. Notice that the existence of parity effects implies that the number of available data points is rather small: for $L \equiv 0(\bmod 4)$ it is two, and for $L \equiv 2(\bmod 4)$, three. Moreover, if we use the improved Ansatz

$$
f_{L}(q,-1)=f_{\text {bulk }}(q,-1)+\frac{G \pi c_{L}(q,-1)}{6 L^{2}}+A L^{-4},
$$

for $L=L_{\min }, L_{\min }+4, L_{\min }+8$, the number of data points $c_{L}(q,-1)$ is further reduced to one and two, respectively (see table 6). In this table, the estimates $\left(q_{\max }(L), c_{\max }(L)\right)$ seem to converge to the expected value $(3,1)$ as $L$ increases. 


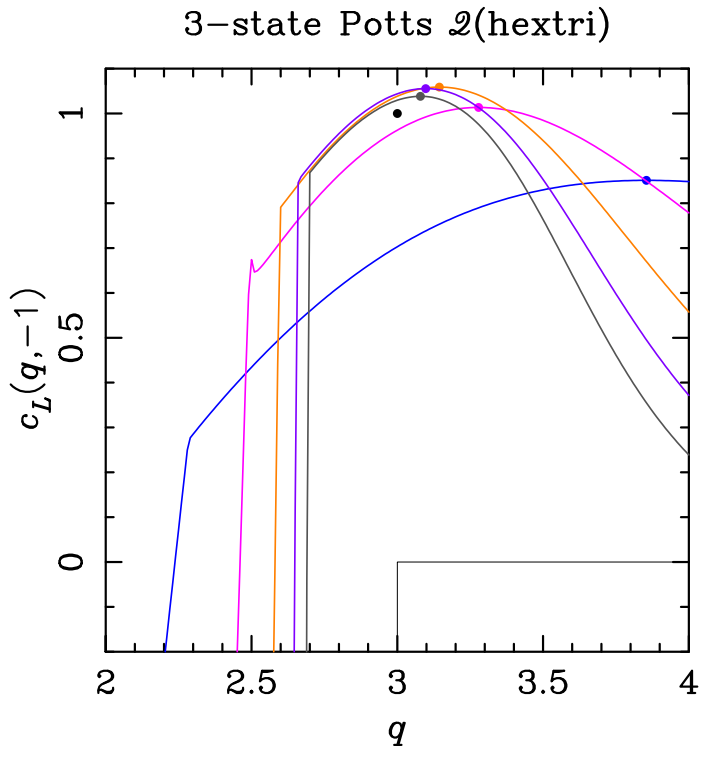

(a)

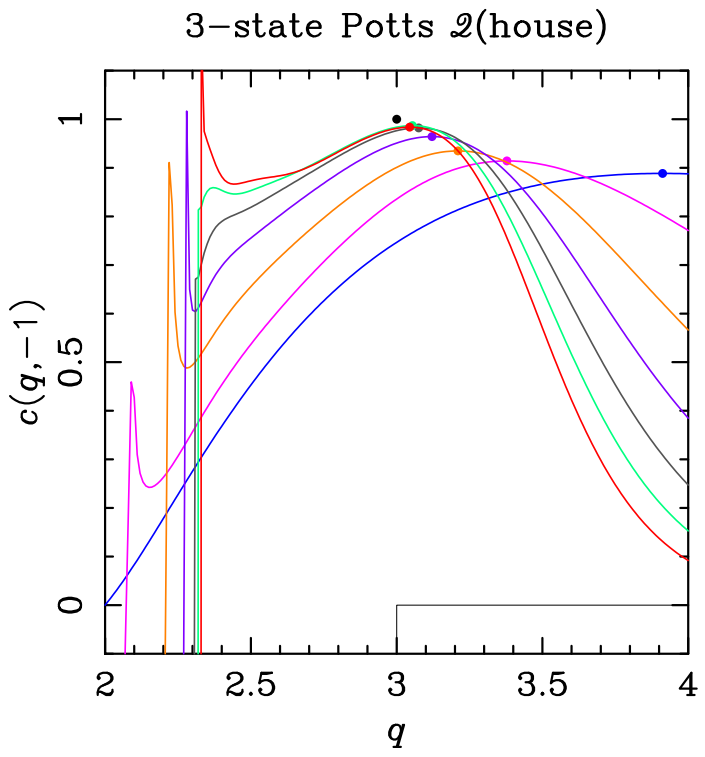

(b)

Figure 10: Estimates of the central charge $c_{L}(q,-1)$ for the 3-state Potts model on the $\mathcal{Q}$ (hextri) (a) and $\mathcal{Q}$ (house) (b) lattices with cylindrical boundary conditions. We have used the Ansatz (3.24) for $L=L_{\min }, L_{\min }+4$ to avoid systematic parity effects. We show the results for $L_{\min }=2$ (blue), $L_{\min }=4$ (pink), $L_{\min }=6$ (orange), $L_{\min }=8$ (violet), $L_{\min }=10$ (dark gray), $L_{\min }=12$ (medium green), and $L_{\min }=14$ (red). The dots at each curve mark the position of the maximum value at $\left(q_{\max }(L), c_{\max }(L)\right)$. The black lines show the expected result for $q>3$, and the black dot at $(q, c)=(3,1)$ shows the result predicted by conjecture 1.1(a).

We can also compute the largest real value of $q$ (if it exists at all!) where the limiting curve $\mathcal{B}_{L}$ crosses the real $q$-axis. This point $q_{c}(L)$ corresponds to two dominant eigenvalues of the TM becoming equimodular. These values are also displayed in table 6. For cylindrical boundary conditions, there is no such point in many cases $[31,32,68]$. This happens for strips of the $\mathcal{Q}$ (hextri) lattice for $L=2$ (in this case the TM is one-dimensional), and $L=4,12$. In these latter cases, the limiting curve has a pair of complex conjugate endpoints rather close to the real $q$-axis. We will use the real part of such endpoints as our estimate for $q_{c}(L)$. As $L$ increases, the estimates for $\operatorname{Re} q_{c}(L)$ seem to grow towards $q_{c}=3$ (from below), but the FSS corrections seem to be larger than for the estimates $q_{\max }(L)$.

Our goal is to consider the limit $L \rightarrow \infty$ of these quantities. We first tried standard power-law fits:

$$
\begin{aligned}
q_{\max }(L) & =q_{c}(\mathcal{Q} \text { (hextri) })+A_{q} L^{-\omega_{q}}, \\
\operatorname{Re} q_{c}(L) & =q_{c}(\mathcal{Q} \text { (hextri) })+B_{q} L^{-\omega_{q}^{\prime}}, \\
c_{\max }(L) & =c\left(q_{c},-1\right)+A_{c} L^{-\omega_{c}},
\end{aligned}
$$




\begin{tabular}{|c|c|c|c|}
\hline \multicolumn{4}{|c|}{ Ansatz (3.24) } \\
\hline$L$ & $q_{\max }(L)$ & $c_{\max }(L)$ & $q_{c}(L)$ \\
\hline 2 & 3.8544146155 & 0.8508786050 & \\
\hline 4 & 3.2788982545 & 1.0133854086 & $1.8286335019 \pm 0.0559426374 i$ \\
\hline 6 & 3.1443621430 & 1.0588380075 & 2.2837808646 \\
\hline 8 & 3.0975518402 & 1.0554325766 & 2.4976597599 \\
\hline 10 & 3.0795627986 & 1.0383006482 & 2.5973897407 \\
\hline 12 & & & $2.6580810405 \pm 0.0007531318 i$ \\
\hline 14 & & & 2.6959102812 \\
\hline \multicolumn{4}{|c|}{ Ansatz (3.25) } \\
\hline$L$ & $q_{\max }(L)$ & $c_{\max }(L)$ & \\
\hline 2 & 3.1224187094 & 1.1074883241 & \\
\hline 4 & 3.0645909602 & 1.0826966910 & \\
\hline 6 & 3.0547444360 & 1.0284408966 & \\
\hline
\end{tabular}

Table 6: Finite-size results for the $\mathcal{Q}$ (hextri) lattice with cylindrical boundary conditions of widths $L$. The second and third columns show the position $q_{\max }(L)$ of the maximum value of the central charge $c_{L}(q,-1)$, and the value $c_{\max }(L)$ attained at this point. These estimates are obtained by fitting the free-energy $f_{L}(q,-1)(3.23)$ using the Ansatz (3.24) on the top part (resp. (3.25) on the bottom part) at values of the width $L, L+4$ (resp. $L, L+4, L+8$ ) to avoid parity effects. The fourth column shows for each strip width $L$, the point(s) $q_{c}(L)$ of the limiting curve $\mathcal{B}_{L}$ closest to the real $q$-axis. The results in the first two columns for the Ansatz (3.24) have already appeared in [51, table II].

as we expect that $\lim _{L \rightarrow \infty} q_{\max }(L)=\lim _{L \rightarrow \infty} q_{c}(L)=q_{c}(\mathcal{Q}$ (hextri)).

Let us start with the estimate for $q_{c}=q_{c}(\mathcal{Q}$ (hextri)). First, we consider the data for $\operatorname{Re} q_{c}(L)$ (red points in figure 11(a)). Due to parity affects, we have fitted the data with $L \equiv 0(\bmod 4)$ and $L \equiv 2(\bmod 4)$ separately to the Ansatz (3.26b). In the former case we obtain $q_{c} \approx 2.84$ and $w_{q}^{\prime} \approx 1.57$ for $L_{\min }=4$, and in the latter, $q_{c} \approx 2.82$ and $\omega_{q}^{\prime} \approx 1.71$ for $L_{\min }=6$. In this latter fit, all data points have no imaginary part; so we tend to trust better this estimate than the other one. If we fix $\omega_{q}^{\prime}=2$ in the last fit, we obtain $q_{c}=2.80(6)$ for $L_{\min }=10$.

Now we consider the values of the estimates $q_{\max }(L)$ obtained with the 2-parameter (2P) Ansatz (3.24) (orange dots in figure 11(a)). In this case, there are three points with $L \equiv 2(\bmod 4)$. The power-law fit $(3.26 a)$ gives $q_{c} \approx 3.04$ and $\omega_{q} \approx 1.86$ for $L_{\min }=2$. If we fix $\omega_{q}=2$ and repeat the fit, we obtain $q_{c}=3.04(4)$ for $L_{\min }=4$.

Finally, we have the estimates $q_{\max }(L)$ obtained via the 3 -parameter (3P) Ansatz (3.25) (dark gray points in figure 11(a)). There are only two data points for $L \equiv 2$ $(\bmod 4)$, so we fix $\omega_{q}=2$ and perform the fit to the Ansatz (3.26a); we obtain $q_{c}=3.05$ for $L_{\min }=2$.

The above estimates look rather similar and close to the expected value $q_{c}=3$. 
Potts model 2(hextri)

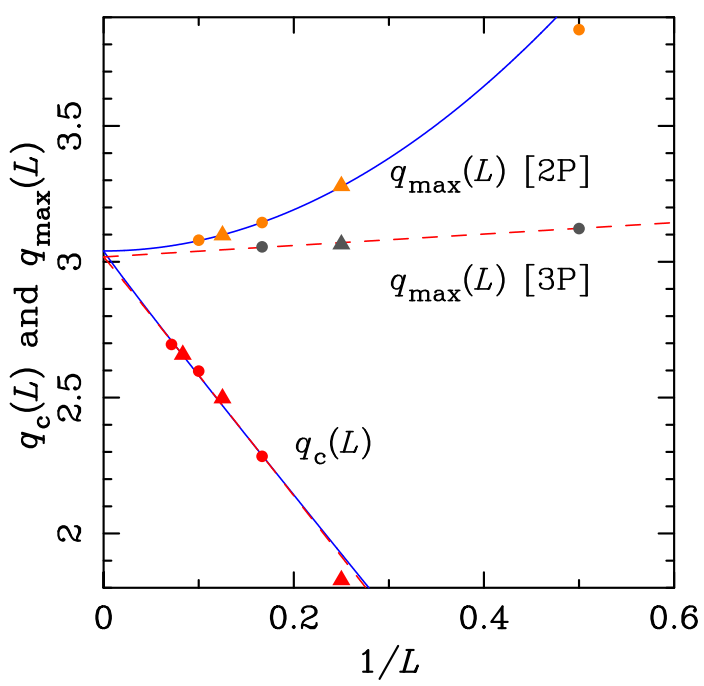

(a)

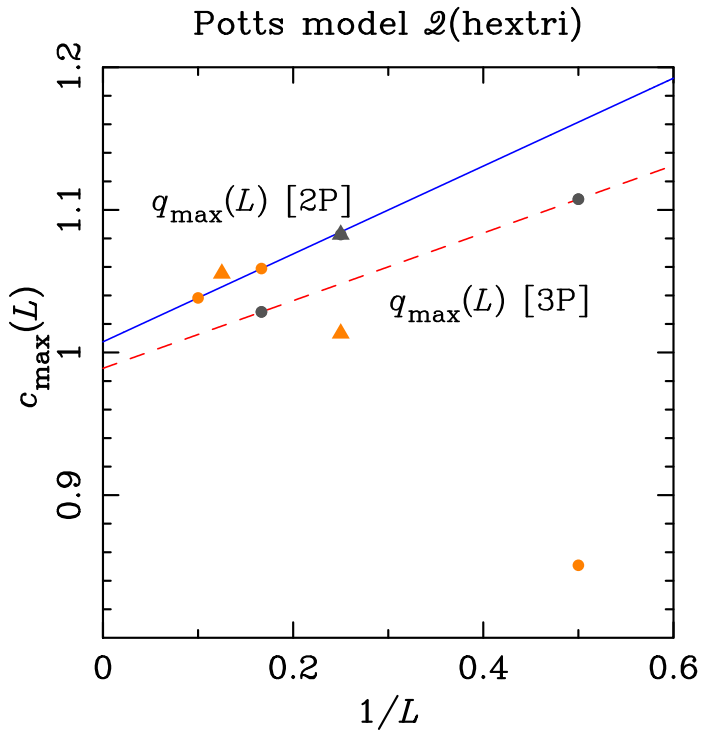

(b)

Figure 11: Fits for the critical parameters of the AF Potts model on the $\mathcal{Q}$ (hextri) lattice with cylindrical boundary conditions. (a) Value of $q_{c}(\mathcal{Q}$ (hextri)). The upper (orange) points points correspond to the estimates of $q_{\max }(L)$ using the Ansatz (3.24), the middle (dark gray) points corresponds to the Ansatz (3.25), and the lower (red) points to those of $\operatorname{Re} q_{c}(L)$ (see table 6$)$. The points corresponding to $L \equiv 2(\bmod 4)$ (resp. $L \equiv 0(\bmod 4))$ are depicted as solid circles $\bullet$ (resp. triangles $\boldsymbol{\Delta})$. The solid blue (resp. dashed red) curves show the simultaneous fit of the data sets $\operatorname{Re} q_{c}(L)$ and $q_{\max }(L)$ obtained by using the Ansatz (3.24) (resp. the Ansatz (3.25)). (b) Value of $c\left(q_{c},-1\right)$. The data points have the same colour/symbol code is as in panel (a) (see table 6). The solid blue (resp. dashed red) curve shows the fit of the data set $c_{\max }(L)$ obtained by using the Ansatz (3.24) (resp. the Ansatz (3.25)).

Moreover, the data set $\operatorname{Re} q_{c}(L)$ seems to increase monotonically as $L$ grows tending to $q_{c}=2.80(6)$, while the data set $q_{\max }(L)$ (either coming from the $2 \mathrm{P}$ or $3 \mathrm{P}$ Ansätze $(3.24) /(3.25)$, respectively) seems to decrease monotonically as $L$ grows tending to $q_{c}=3.04(4)$. The expected value $q_{c}=3$ is just in between these two estimates (but many standard deviations away!). If we fit these two estimates to a constant, we get $q_{c}=2.97(3)\left(\right.$ with $\chi^{2}=11.08,1 \mathrm{DF}$, and $\left.\mathrm{CL}=0.087 \%\right)$.

We might obtain an a priori better estimate if we try to fit several data sets together with a single Ansatz:

$$
q_{c}(L)=q_{c}(\mathcal{Q} \text { (hextri) })+ \begin{cases}C_{1} L^{-\omega_{1}} & \text { if data is } \operatorname{Re} q_{c}(L), \\ C_{2} L^{-\omega_{2}} & \text { if data is } q_{\max }(L) .\end{cases}
$$

We have used Mathematica's NonlinearModelFit function to perform this more complex fit. If we consider the data sets $\operatorname{Re} q_{c}(L)$ and $q_{\max }(L)$ coming from the $2 \mathrm{P}$ Ansatz $(3.24)$ for $L \equiv 2(\bmod 4)$, and fix $\omega_{2}=2$ (as we did above), we 
find $q_{c}=3.04(3)$ and $\omega_{1}=0.97(10)$ for $L_{\min }=6$. A better determination could be obtained by fixing $\omega_{1}=1$ : the result is $q_{c}=3.03(2)$ for $L_{\min }=6$.

If we repeat the same procedure with the data sets $\operatorname{Re} q_{c}(L)$ and $q_{\max }(L)$ coming from the 3P Ansatz $(3.25)$ for $L \equiv 2(\bmod 4)$, the slow variation of $q_{\max }(L)$ versus $1 / L$ suggests to fix $\omega_{1}=1$ in Ansatz (3.27). Then we get $q_{c}=3.02(3)$ and $\omega_{1}=1.01(10)$ for $L_{\min }=2$. If we further fix $\omega_{1}=1$, we obtain $q_{c}=3.02(2)$.

Notice that we refrain from merging both data sets for $q_{\max }(L)$ (i.e., using Ansätze $(3.24) /(3.25))$, as they are not statistically independent. The curves obtained in the last two combined fits are depicted in figure 11(a). Moreover, the two distinct curves fitting the same data set $\operatorname{Re} q_{c}(L)$ are actually almost identical. In conclusion, our preferred estimated for the combined Ansatz (3.27) is $q_{c}=3.03(2)$.

If we merge the estimates $q_{c}=2.97(3)$ and $q_{c}=3.03(2)$, we obtain

$$
q_{c}(\mathcal{Q}(\text { hextri }))=3.00(2)
$$

with $\chi^{2}=1.92,1 \mathrm{DF}$, and CL $=17 \%$. This result is in full agreement with conjecture 1.1(a). Indeed, the error bar should be taken with caution.

Figure 11(b) shows the data points for the quantity $c_{\max }(L)$. It is clear that both the parity effects and the FSS corrections are rather strong in this case, while the number of data points is very small. Therefore, estimating the value of $c(3,-1)$ would be very difficult.

We have first considered the points $c_{\max }(L)($ for $L \equiv 2(\bmod 4))$ obtained with the 2P Ansatz (3.24) (orange dots in figure 11(b)). If we fix $\omega_{c}=1$ in the Ansatz (3.26c), we get a sensible fit $c(3,-1) \approx 1.01$ for $L_{\min }=6$ (solid blue curve in figure $\left.11(\mathrm{~b})\right)$. If we add a second term $B L^{-2}$ to this Ansatz, we get $c(3,-1) \approx 0.97$ for $L_{\min }=2$.

If we consider the estimates $c_{\max }(L)$ obtained with the improved $3 \mathrm{P}$ Ansatz (3.25) (dark gray points in figure 11(b)), we can fit them to the Ansatz (3.26c) by fixing $\omega_{c}=1$. We get $c(3,-1) \approx 0.99$ for $L_{\min }=2$ (dashed red curve in figure $11(\mathrm{~b})$ ).

If we consider all of the above, we conclude that our final estimate is

$$
c(3,-1)=0.99(2)
$$

where the error bar should be taken with a grain of salt, as it takes into account the range of the few estimates discussed above. Again this results agrees well with conjecture 1.1(a).

\subsubsection{Transfer matrix for $\mathcal{Q}$ (house)}

We have repeated the previous computation for the $\mathcal{Q}$ (house) lattice. In order to build this lattice, we have used the representation depicted in figure 12. Again, the width of the strip should be an even integer $L$. If we label the vertices on the bottom row of a strip width of size $L$ as $1,2, \ldots, L$, as in figure 12, then the TM for $\mathcal{Q}$ (house) reads:

$$
\mathrm{T}=\mathrm{V}_{0}^{(\text {odd })} \cdot \mathrm{H} \cdot \mathrm{V}_{0}^{(\text {even })} \cdot \mathrm{H} \cdot \mathrm{V} \cdot \mathrm{H}
$$


where the operators $\mathrm{H}, \mathrm{V}$ and $\mathrm{V}_{0}^{(\text {even) }}$ are given by $(3.20) /(3.22)$, and $\mathrm{V}_{0}^{(\text {odd })}$ is expressed as

$$
\mathrm{V}_{0}^{(\text {odd })}=\prod_{i=1}^{L / 2} \mathrm{D}_{2 i-1}
$$

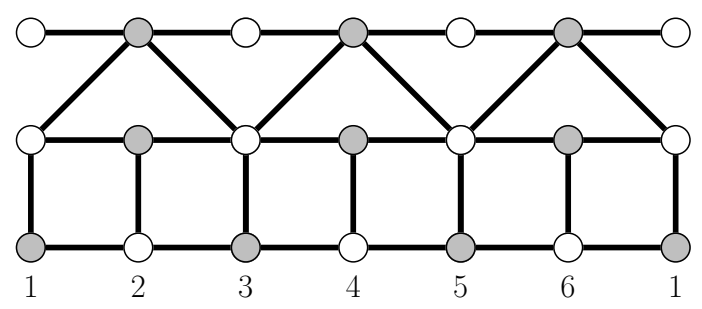

Figure 12: TM construction for a layer of a strip graph of the $\mathcal{Q}$ (house) lattice of width $L=6$ and cylindrical boundary conditions. There are two classes of nonequivalent vertices, depicted respectively, as white and gray dots.

The space of connectivities is the same as for the $\mathcal{Q}$ (hextri) lattice. We have also implemented the symbolic computation of the TM by a PERL script for widths $2 \leq L \leq 16$. It is worth noticing that we found many rows that were exactly zero in all $\mathrm{TM}$ for $L \geq 4$. In fact, these rows corresponded to connectivities for which some of the odd-labelled sites were not singletons. Therefore, we could consider the smaller subspace of connectivities in which all odd-labelled sites are singletons. The dimensions of this 'improved' TM are given by by 1, 2, 3, 6, 10, 24, 49, and 130 for $L=2,4, \ldots, 16$. Indeed, we have checked the results by computing symbolically the chromatic polynomial (using both TM) for strip graphs of size $L \times N$ with $2 \leq N \leq 20$, and compare them to the results provided by other programs $[6,21]$. The agreement is also perfect.

For each width $L$, we have computed the free energy $f_{L}(q,-1)$ in the interval $q \in[2,4]$ in terms of the dominant eigenvalue $\lambda_{*}$ of the corresponding TM (see (3.23)). The central charge is then estimated by using the Ansatz (3.24), where for this lattice the geometrical factor is $G=2 \sqrt{3}$ (see appendix A). Following the same procedure as in the previous section, we obtain the results displayed in figure 10(b).

It is clear from this figure that, for each value $L=L_{\text {min }}$, the central charge presents a maximum $c_{\max }(L)$ at $q=q_{\max }(L)$. These values are shown in table 7 . Notice that the estimates for $q_{\max }(L)$ seem to converge rather quickly to the expected value $q_{c}=3$ from above, while the estimates for $c_{\max }(L)$ seem also to increase as $L$ increases. A FSS study is needed to tell whether these estimates converge to the value $c=1$ predicted by conjecture 1.1(a).

We find that there is an endpoint of the limiting curve for $L=4$ very close to the real $q$-axis. But contrary to what happens for the $\mathcal{Q}$ (hextri) lattice, for $6 \leq L \leq 14$, we find that the limiting curve $\mathcal{B}_{L}$ contains an interval on the real $q$-axis. As $L$ increases, this interval moves to larger values of $q$ and its length decreases. It varies from $q \approx[2.056685,2.096902]$ for $L=6$ to $q \approx[2.329251,2.330336]$ for $L=16$. 


\begin{tabular}{rccl}
\hline \hline \multicolumn{4}{c}{ Ansatz $(3.24)$} \\
\hline$L$ & $q_{\max }(L)$ & $c_{\max }(L)$ & \multicolumn{1}{c}{$q_{c}(L)$} \\
\hline 2 & 3.7094424595 & 0.8808411388 & \\
4 & 3.3184514005 & 0.9155222019 & $1.7562125170 \pm 0.0575636262 i$ \\
6 & 3.1770041039 & 0.9424270745 & 2.0770205951 \\
8 & 3.1034254588 & 0.9699972659 & 2.2109211636 \\
10 & 3.0667634439 & 0.9837482525 & 2.2721580696 \\
12 & 3.0500783475 & 0.9855564633 & 2.3029952390 \\
14 & & & 2.3198098469 \\
16 & \multicolumn{4}{c}{ Ansatz $(3.25)$} & 2.3297948774 \\
\hline \multicolumn{4}{c}{$c_{\max }(L)$} \\
\hline$L$ & $q_{\max }(L)$ & \\
\hline 2 & 3.1556222914 & 0.9623949139 \\
4 & 3.0556456566 & 1.0064534361 \\
6 & 3.0191585619 & 1.0209664138 \\
8 & 3.0174550444 & 1.0040970911 \\
\hline \hline
\end{tabular}

Table 7: Finite-size results for the $\mathcal{Q}$ (house) lattice with cylindrical boundary conditions of widths $L$. The second and third columns show the position $q_{\max }(L)$ of the maximum value of the central charge $c_{L}(q,-1)$, and the value $c_{\max }(L)$ attained at this point. These estimates are obtained by fitting the free-energy $f_{L}(q,-1)(3.23)$ using the Ansatz (3.24) on the top part (resp. (3.25) on the bottom part) at values of the width $L, L+4$ (resp. $L, L+4, L+8$ ) to avoid parity effects. The fourth column shows for each strip width $L$, the point(s) $q_{c}(L)$ of the limiting curve $\mathcal{B}_{L}$ closest to the real $q$-axis (see text).

Therefore, we will not consider this estimate to try to compute $q_{c}$ for this lattice. The values displayed in table 7 correspond to the point in that interval where the limiting curve emerges from the real $q$-axis.

As for the $\mathcal{Q}$ (hextri) lattice, we expect parity effects. To obtain the estimate for $q_{c}=q_{c}(\mathcal{Q}$ (house) $)$, we first consider the data $q_{\max }(L)$ coming from the $2 \mathrm{P}$ Ansatz (3.24) (orange points in figure 13(a)). A power-law fit (3.26a) gives $q_{c} \approx 2.97$ for $L_{\min }=4$ for data with $L \equiv 0(\bmod 4)$, and the estimate $q_{c} \approx 3.02$ for $L_{\min }=6$ for data with $L \equiv 2(\bmod 4)$.

If we perform similar fits for the data $q_{\max }(L)$ coming from the 3P Ansatz (3.24) (dark gray points in figure 13(a)), we obtain the estimates $q_{c} \approx 3.02$ for $L_{\min }=4$, and $q_{c} \approx 3.01$ for $L_{\min }=2$, respectively.

The lack of many data points discourage us to try to give an 'objective' error bar. By inspecting the above results, we conclude that our best estimate is

$$
\left.q_{c}(\mathcal{Q} \text { (house })\right)=2.99(3),
$$

in agreement with conjecture 1.1(a). Again, the error bar should be taken with care. 


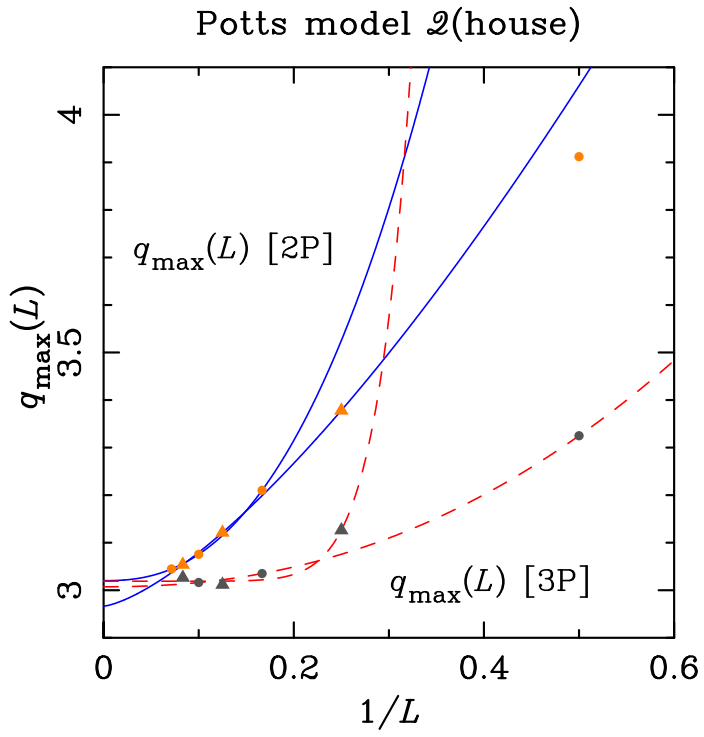

(a)

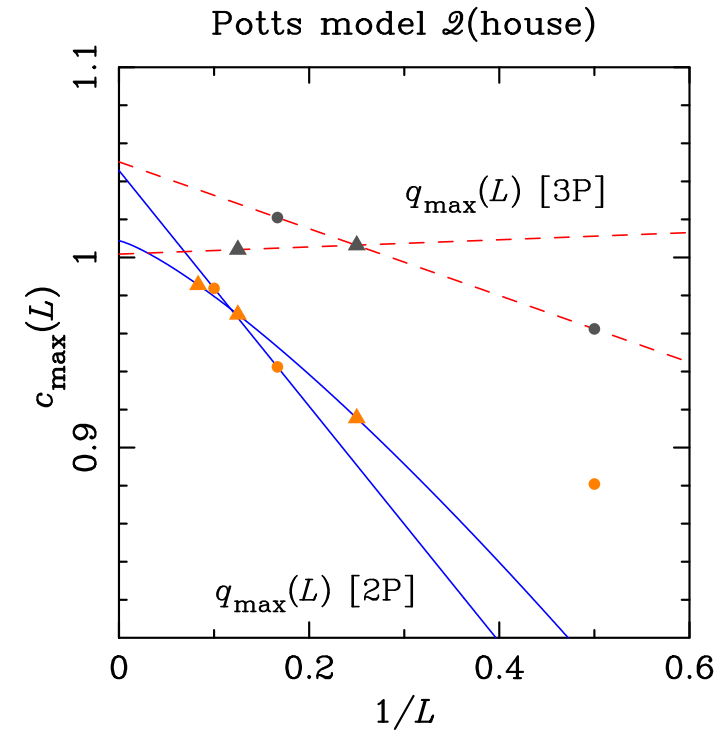

(b)

Figure 13: Fits for the critical parameters of the AF Potts model on the $\mathcal{Q}$ (house) lattice with cylindrical boundary conditions. (a) Value of $q_{c}(\mathcal{Q}$ (house)). The upper (orange) points points correspond to the estimates of $q_{\max }(L)$ using the Ansatz (3.24), and the lower (dark gray) points corresponds to the Ansatz (3.25). (see table 7). The points corresponding to $L \equiv 2(\bmod 4)($ resp. $L \equiv 0(\bmod 4))$ are depicted as solid circles - (resp. triangles $\mathbf{\Delta}$ ). The solid blue (resp. dashed red) curves show the fits of the data set $q_{\max }(L)$ obtained by using the $2 \mathrm{P}$ Ansatz (3.24) (resp. the 3P Ansatz (3.25)). (b) Value of $c\left(q_{c},-1\right)$. The data points and curves have the same colour/symbol code is as in panel (a) (see table 7).

The estimate for $c(3,-1)$ can be obtained by considering the data $c_{\max }(L)$ coming from the $2 \mathrm{P}$ Ansatz (3.24) (orange points in figure 13(b)). A power-law fit (3.26a) gives $c(3,-1) \approx 1.01$ for $L_{\min }=4$ and $c(3,-1) \approx 1.05$ for $L_{\min }=6$ (after fixing $w_{c}=1$, because the power-law fit does not converge).

If we use the data $q_{\max }(L)$ coming from the $3 \mathrm{P}$ Ansatz (3.24) (dark gray points in figure $13(\mathrm{~b})$ ), we obtain the estimates $c(3,-1) \approx 1.05$ for $L_{\min }=2$, and $c(3,-1) \approx$ 1.00 for $L_{\min }=4$.

Finally, our 'best' estimate is given by

$$
c(3,-1)=1.03(3)
$$

in agreement with conjecture 1.1(a).

\subsection{Critical polynomials}

The location of the phase transitions for the quadrangulations in figures 2 and 3 can also be studied by the method of critical polynomials $[28,35,36,70,71]$. These 
polynomials $P_{B}(q, v)$ can in principle be computed for any lattice generated by the tessellation of the plane by some finite basis $B$. However, we wish here to take advantage of the efficient TM algorithm of [28] which supposes that $B$ admits a so-called four-terminal representation [28, Section 3.4].

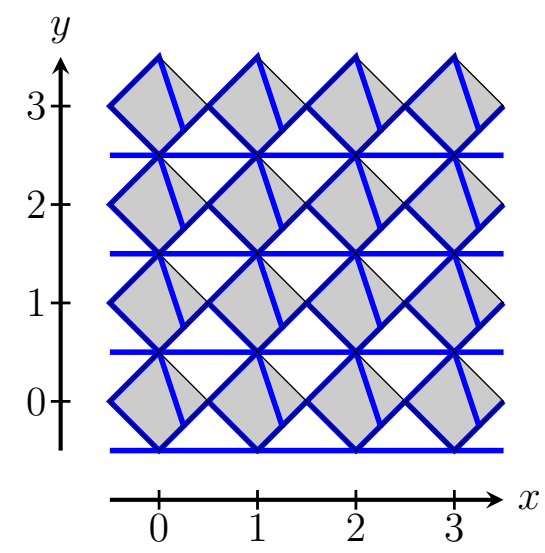

Figure 14: Four-terminal representation of the quadrangulation $\mathcal{Q}$ (hextri).

A four-terminal representation of $\mathcal{Q}$ (hextri) is shown in figure 14, for a basis of size $4 \times 4$ elementary cells. In general, a four-terminal representation is a checkerboard alternation of gray and white squares, such that the white squares are either empty or contain at most diagonally a horizontal edge from $B$, whereas all the remaining vertices and edges from $B$ reside within the gray squares. The most efficient computations are obtained when the number of edges and vertices per gray square is as large as possible. The representation in figure 14 contains 6 edges and 3 vertices per gray square (this count includes by convention the diagonals in the white squares), which can be compared with [28, table 3].

Once a four-terminal representation for the lattice of interest has been found, $P_{B}(q, v)$ can be computed by relatively minor modifications of the existing algorithm [28]. It suffices to express the content within each gray square as an operator $\check{\mathrm{R}}_{i}[28$, section 3.4] which, in turn, is written as a product of elementary operators $\mathbf{H}_{j}, \mathbf{V}_{j}$ and $\mathrm{E}_{j}$. The first two of these, $\mathrm{H}_{j}$ (resp. $\mathrm{V}_{j}$ ), correspond to the insertion of an edge of $B$ in the horizontal (resp. vertical) time direction, with the usual convention that each gray square is traversed in the North-East direction under the time evolution that defines the transfer matrix. The last operator, $\mathrm{E}_{j}$, is a Temperley-Lieb generator that can be used in some cases (we do not need it here) to accomplish the basic join and detach operations.

For $\mathcal{Q}$ (hextri) we can read off from figure 14 that

$$
\check{\mathrm{R}}_{i}=\mathrm{V}_{i+2} \cdot \mathrm{H}_{i+1} \cdot \mathrm{V}_{i+2} \cdot \mathrm{V}_{i} \cdot \mathrm{H}_{i+1},
$$

which can be compared with the many examples given in [28, Section 4]. In addition, there is a diagonal edge on each white square. 
In general, $\check{\mathrm{R}}_{i}$ is not necessarily given by the same expression for each gray square, and when this is not the case we will typically have an $n \times m$ pattern of different gray squares, with some periodicity in the horizontal and vertical directions. As $B$ must be considered with implicit doubly periodic boundary conditions, for the tiling of the whole plane to be valid this alternation will impose parity constraints on $n$ and/or $m$, and in practice limit the number of sizes that can be studied. For $\mathcal{Q}$ (hextri) the expression for $\check{\mathrm{R}}_{i}$ is homogeneous, and we can in particular consider bases of size $n \times n$ for any parity of $n$.

The critical polynomial for $\mathcal{Q}$ (hextri) with a $1 \times 1$ basis is readily found as

$$
P_{B}(q, v)=-q^{3}-5 q^{2} v-8 q v^{2}+2 q v^{3}+12 v^{4}+6 v^{5}+v^{6} .
$$

By modifying the algorithm of [28] we have similarly computed $P_{B}(q, v)$ for $n \times n$ bases with $n=1,2,3,4$. They are polynomials of degree $3 n^{2}$ in $q$, and $6 n^{2}$ in $v$. Setting $q=3$, we report in table 8 the unique real zero of $P_{B}(3, v)$ in the ferromagnetic (FM) regime $(v>0)$, along with any zero in the physical part of the AF regime $(-1 \leq v<0)$. There are other real roots in the unphysical AF regime $(v<-1)$ that we do not report in table 8 .

\begin{tabular}{l|c|c}
\hline \hline$n$ & $v_{\mathrm{c}}^{\mathrm{FM}}(n)$ & $v_{\mathrm{c}}^{\mathrm{AF}}(n)$ \\
\hline 1 & 1.53528140860819381729 & \\
2 & 1.53506577625594900753 & -0.91138737767231613799 \\
3 & 1.53500770075339075633 & \\
4 & 1.53499345908015323800 & -0.92997036994718707983 \\
\hline$\infty$ & $1.534987(1)$ & \\
\hline \hline
\end{tabular}

Table 8: Real roots of $P_{B}(q, v)$, to 20 digit numerical precision, for the quadrangulation $\mathcal{Q}$ (hextri). We show the (unique) real roots, $v_{\mathrm{c}}^{\mathrm{FM}}(n)$ and $v_{\mathrm{c}}^{\mathrm{AF}}(n)$, in the intervals $v>0$ and $-1 \leq v<0$ respectively, for the $n \times n$ bases of figure 14 . The latter root only exists for even $n$. The last row labeled ' $\infty$ ' shows our extrapolation as $n \rightarrow \infty$.

The FM zero $v_{\mathrm{c}}^{\mathrm{FM}}(n)$ converges rapidly to the critical coupling $v_{\mathrm{c}}$-a phenomenon which was already studied extensively in [28] for a variety of lattices. A power-law fit

$$
v_{c}^{\mathrm{FM}}(n)=v_{c}^{\mathrm{FM}}+A n^{-\Delta}
$$

for three consecutive data points (i.e., $n=n_{\min }, n_{\min }+1, n_{\min }+2$ ) gives the estimate $v_{c}^{\mathrm{FM}}=1.53498(6)$ for $n_{\min }=2$. If we use Monroe's [55] implementation of the Bulirsh-Stoer [9] extrapolation scheme (MBS) with powers in the range 2.1-3.2, we obtain a more precise result:

$$
v_{\mathrm{c}}^{\mathrm{FM}}=1.534987(1) .
$$

The physical AF zero $v_{\mathrm{c}}^{\mathrm{AF}}(n)$ only exists for even $n$ and appears to converge to the expected critical point, $v_{\mathrm{c}}^{\mathrm{AF}}=-1$, at a much slower rate. This contrasts with 
the results of [28], where $P_{B}(q, v)$ was seen to factorise in exactly solvable cases, thus giving one or more roots $v_{\mathrm{c}}$ with no $n$-dependence at all. In the present case, $P_{B}(3, v)$ does not factorise over the integers for any $n=1,2,3,4$. On the other hand, our conviction that there is a critical point exactly at $v_{\mathrm{c}}^{\mathrm{AF}}=-1$ does not necessarily imply that this model would be exactly solvable, in the sense that (for instance) the corresponding free energy could be computed exactly in the thermodynamic limit.

We have also found four-terminal representations for two of the other quadrangulations of self-dual type in figure 2: namely, $\mathcal{Q}$ (house) and $\mathcal{Q}$ (martini-B). In both cases the operator $\check{\mathrm{R}}_{i}$ depends on the coordinates $(x, y)$ of the gray squares, see figure 14 .

For $\mathcal{Q}$ (house) we have

$$
\check{\mathrm{R}}_{i}= \begin{cases}\mathrm{H}_{i+1} \cdot \mathrm{V}_{i+2} \cdot \mathrm{V}_{i} \cdot \mathrm{H}_{i+1} & \text { for } x \in 2 \mathbb{N}, \\ \mathrm{H}_{i+1} \cdot \mathrm{E}_{i} \cdot \mathrm{H}_{i+1} \cdot \mathrm{V}_{i+2} & \text { for } x \in 2 \mathbb{N}+1,\end{cases}
$$

and there are horizontal diagonals in the white squares with $x \in 2 \mathbb{N}+\frac{1}{2}$. This representation is well-defined for $n$ even, and it has 4 edges and 2 vertices per gray square. We have computed $P_{B}(q, v)$ for this lattice using $n \times n$ bases with $n=2,4$. The former case does not have any root in the physical AF regime. However, for $n=4$ we find a root at $v_{\mathrm{c}}^{\mathrm{AF}}(4)=-0.94524856890048286949 \cdots$, which is reasonably close, but not exactly equal, to $v_{\mathrm{c}}^{\mathrm{AF}}=-1$.

For $\mathcal{Q}($ martini-B) we have found

$$
\check{\mathrm{R}}_{i}= \begin{cases}\mathrm{H}_{i+1} \cdot \mathrm{V}_{i+2} \cdot \mathrm{V}_{i} \cdot \mathrm{H}_{i+1} & \text { for } x \in 4 \mathbb{N}, \\ \mathrm{H}_{i+1} \cdot \mathrm{V}_{i+2} \cdot \mathrm{V}_{i} \cdot \mathrm{H}_{i+1} \cdot \mathrm{V}_{i} & \text { for } x \in 4 \mathbb{N}+1, \\ \mathrm{~V}_{i} \cdot \mathrm{E}_{i+1} \cdot \mathrm{E}_{i+2} \cdot \mathrm{H}_{i+1} & \text { for } x \in 4 \mathbb{N}+2, \\ \mathrm{H}_{i+1} \cdot \mathrm{E}_{i} \cdot \mathrm{H}_{i+1} \cdot \mathrm{V}_{i+2} & \text { for } x \in 4 \mathbb{N}+3,\end{cases}
$$

and there are again horizontal diagonals in the white squares with $x \in 2 \mathbb{N}+\frac{1}{2}$. This representation is well-defined only for $n \in 4 \mathbb{N}$ and has 4 edges per gray square.

We now turn to the non-self-dual quadrangulations of figures $3-4$. The fact that most of these involve vertices of high degree has prevented us from finding fourterminal representations. There is of course no doubt that some TM formulation could be found for those lattices, but we refrain from studying this issue further here, since it would involve a more profound rewriting of the algorithm given in [28].

The first of the non-self-dual quadrangulations, $\mathcal{Q}$ (diced), has in fact already been treated in [28, section 4.10] where a four-terminal representation and explicit polynomials $P_{B}(q, v)$ for several $n \times n$ bases were given. To find the corresponding AF critical point, we shall instead resort to the eigenvalue method of [29], which amounts to finding numerically the relevant root $v_{\mathrm{c}}^{\mathrm{AF}}(n)$ of $P_{B}(3, v)$ for a basis of size $n \times m$, directly in the limit $m \rightarrow \infty$. This TM method has the advantage over that of [28] that the size $n$ can be doubled with approximately the same computational effort.

The results are shown in table 9. If we fit the data for $v_{\mathrm{c}}^{\mathrm{FM}}(n)$ to the power-law fit (3.36) using three consecutive data points (i.e., $n=n_{\min }, n_{\min }+2, n_{\min }+4$ ), we obtain a rather accurate fit for $n_{\min }=8$ :

$$
v_{\mathrm{c}}^{\mathrm{FM}}=1.600919708(6),
$$




\begin{tabular}{r|c|c}
\hline \hline$n$ & $v_{\mathrm{c}}^{\mathrm{FM}}(n)$ & $v_{\mathrm{c}}^{\mathrm{AF}}(n)$ \\
\hline 2 & 1.60090047247076387216 & -0.93449469491145567949 \\
4 & 1.60092569962114202789 & -0.93889690618313817225 \\
6 & 1.60092045368358355317 & -0.93976678350525022210 \\
8 & 1.60091983209526681674 & -0.94017098791714722205 \\
10 & 1.60091973715419384611 & -0.94038789257375557598 \\
12 & 1.60091971679606195523 & -0.94051494788357303489 \\
\hline$\infty$ & $1.600919708(6)$ & $-0.94080(1)$ \\
\hline \hline
\end{tabular}

Table 9: Real roots of $P_{B}(q, v)$, to 20 digit numerical precision, for the quadrangulation $\mathcal{Q}$ (diced). We show the (unique) real roots, $v_{\mathrm{c}}^{\mathrm{FM}}(n)$ and $v_{\mathrm{c}}^{\mathrm{AF}}(n)$, in the intervals $v>0$ and $-1 \leq v<0$ respectively, for $n \times \infty$ bases. They are obtained from the eigenvalue method of [29]. The last row labeled ' $\infty$ ' shows our extrapolations as $n \rightarrow \infty$. The results for the AF critical point $v_{\mathrm{c}}^{\mathrm{AF}}(n)$ have already appeared in [51, table IV].

where the error is twice the difference between the estimates with $n_{\min }=6,8$. The convergence is rather fast, as the exponent $\Delta$ varies from $\Delta \approx 6.2$ for $n_{\min }=6$ to $\Delta \approx 6.5$ for $n_{\min }=8$. If we use the MBS extrapolation scheme with powers in the range 5.6-7.5, we obtain a slightly less precise result:

$$
v_{\mathrm{c}}^{\mathrm{FM}}=1.60092049(1) \text {. }
$$

The convergence for the AF critical point is slower. If we do the same procedure with the data in the third column in table 9 , we find an estimate

$$
v_{\mathrm{c}}^{\mathrm{AF}}=-0.9410 \pm 0.0002,
$$

with the error bar estimated as twice the difference between the estimates for $n_{\min }=$ 6,8 . In this case the value of the exponent $\Delta$ stays around $\Delta \approx 1.4-1.6$. We performed the MBS algorithm with powers in the range 1.2-1.5, and obtained a more precise result:

$$
v_{\mathrm{c}}^{\mathrm{AF}}=-0.94080(1) .
$$

This value is compatible with (and more precise than) the MC result shown in Table 3: $v_{\mathrm{c}}^{\mathrm{AF}}=-0.94075(12)$.

Notice that for the $\mathcal{Q}$ (diced) we have obtained estimates for both the FM and AF regimes, and the convergence is a lot faster and the result more precise when the corresponding critical theory is in the FM Potts universality class, rather than in the $\mathrm{AF}$ one.

\section{Discussion}

In this paper we have proposed a conjecture (conjecture 1.1) that predicts the phase diagram of the 3 -state Potts AF model on any plane quadrangulation. This 
phase diagram depends on a single characteristic of these quadrangulations: namely, if they are of self-dual or non-self-dual type. Notice that this prediction deals with an infinite set of models. This conjecture follows from general arguments based on the height representation of these models at zero temperature, and on universality. Indeed, it generalises previous studies on two particular models: the square- $[10,67]$ and the diced-lattice [45] cases.

We have tested the predictions of conjecture 1.1 on four quadrangulations of selfdual type (see figure 2), and on seven quadrangulations of non-self-dual type (see figures 3-4). We have made extensive MC simulations on all of these quadrangulations, as well as detailed TM computations on two of them (i.e., $\mathcal{Q}$ (hextri) and $\mathcal{Q}$ (house)) and $\mathrm{CP}$ computations for $\mathcal{Q}$ (hextri), $\mathcal{Q}$ (house), $\mathcal{Q}$ (martini-B), and $\mathcal{Q}$ (diced). All these results are in good agreement with the predictions of conjecture 1.1. In table 10 we display all the quadrangulations $\Gamma$ considered in this work. We also include the two well-known cases studied in the literature: the square [10,67] and the diced [45] lattices.

\begin{tabular}{|c|cc|c|}
\hline $\begin{array}{c}\text { Quadrangulation } \\
\Gamma\end{array}$ & Sublattice & Sublattice & $v_{c}(3)$ \\
$G_{0}$ & $G_{1}$ & \\
\hline \multicolumn{4}{|c|}{ Quadrangulations of self-dual type } \\
\hline square & square & square & -1 \\
$\mathcal{Q}$ (hextri) & hextri & hextri & -1 \\
$\mathcal{Q}$ (house) & house & house & -1 \\
$\mathcal{Q}$ (martini-B) & martini-B & martini-B & -1 \\
$\mathcal{Q}($ cmm-pmm $)$ & cmm-pmm & cmm-pmm & -1 \\
\hline
\end{tabular}

Quadrangulations of non-self-dual type, $G_{0}$ or $G_{1}$ triangulation [46]

\begin{tabular}{|c|cc|l|}
\hline diced & triangular & hexagonal & $-0.860599(4)$ \\
$\mathcal{Q}$ (martini) & dual martini & martini & $-0.77454(6)$ \\
$\mathcal{Q}$ (cross) & cross & bisected hexagonal & $-0.80057(4)$ \\
$\mathcal{Q}($ asanoha $)$ & asanoha & $3-12$ & $-0.72033(3)$ \\
$G_{2}^{\prime \prime}[24]$ & union-jack & four-eight & $-0.82278(4)$ \\
\hline \multicolumn{4}{|c|}{ Quadrangulations of non-self-dual type, $\boldsymbol{G}_{\mathbf{0}}$ or $\boldsymbol{G}_{\mathbf{1}}$ quadrangulation } \\
\hline $\mathcal{Q}$ (diced) & diced & kagome & $-0.94075(12)$ \\
$\mathcal{Q}($ ruby $)$ & dual ruby & ruby & $-0.95588(9)$ \\
$G_{3}^{\prime \prime}[24]$ & $G_{2}^{\prime}$ & decorated four-eight & $-0.72278(2)$ \\
\hline
\end{tabular}

Table 10: Some plane quadrangulations $\Gamma$ arising from a dual pair $\left(G_{0}, G_{1}\right)$. For each of them, we display the critical temperature $v_{c}(3)$ for the 3-state AF Potts model on $\Gamma$. The square $[10,67]$ and diced $[45]$ lattices are added for completeness.

Even though we have found an excellent empirical support for conjecture 1.1, there are a few issues (already raised in the text) that might limit the generality of this conjecture: 
- For the quadrangulations of self-dual type, we find that the existence of a zerotemperature critical point depends on the fact that the stiffness of the effective Gaussian field theory satisfies $K<K_{c}=\pi / 2$. Even though we have seen that this is the case for the four quadrangulations of this type considered in this work, we have found that the value of $K$ grows smoothly with the maximum degree $\Delta$ of the quadrangulation. Notice that the study of $\mathcal{Q}(\mathrm{cmm}-\mathrm{pmm})$ with $\Delta=8$ was motivated to test whether increasing $\Delta$ would produce or not a large increment in $K$; but the answer was negative. However, it is still possible that for quadrangulations of this type with $\Delta$ large enough, $K>K_{c}$. This would imply that conjecture 1.1(a) does not hold for such quadrangulations. On the other hand, the solution I found in [13] seems to give additional theoretical support to our conjecture.

- For quadrangulations of non-self-dual type, we find that at $T=0$ there are three coexisting (ordered) ideal states (= phases). This implies that there should be some finite-temperature phase transition separating the ordered phase from the disordered high-temperature one. Even though, we have found that in all cases, this phase transition is of second order, we cannot rule out on general grounds the existence of a first-order transition (like in the one undergone by the 3-state AF Potts model on the triangular lattice [1]).

- The argument leading to conjecture 1.1(b) assumes that one sublattice has more vertices than the other one (i.e. $\left|V_{0}\right|<\left|V_{1}\right|$ ). This line of reasoning might be false when both sublattices have the same number of vertices. This observation led us to study carefully two such cases, $\mathcal{Q}$ (diced) and $\mathcal{Q}$ (ruby). We have seen that the fluctuations around the 'naive' ideal states are enough to distinguish between the two sublattice: the one that contains the vertices with largest degree is the ordered one, while the other one is disordered. However, the FM ordering of the former sublattice is only partial, contrary to what happens for the more generic case $\left|V_{0}\right|<\left|V_{1}\right|$.

These questions motivate the study of more complicated lattices in order to find out whether there are any counter-examples to conjecture 1.1.

As a side-effect of the MC computations, we have found that there is also extensive empirical support for conjecture 1.2, which predicts the dynamic critical behavior of the WSK algorithm for the whole class of the 3-state AF Potts models on any quadrangulation. The most important part, in our view, is conjecture 1.2(a), which predicts the total absence of CSD for quadrangulations of self-dual type. Quite often, the WSK algorithm (as well as its SW counterpart for FM models) 'only' reduces the characteristic dynamic critical exponent $z_{\text {int }} \approx 2$ for single-site algorithms to some smaller value; but does not completely eliminate CSD. The only exception known in the literature was the square-lattice 3-state AF model [16,67]. Conjecture 1.2(a) implies that this algorithm eliminates CSD for an infinite class of models. On the other hand, for quadrangulations of non-self-dual type, conjecture 1.2(b) predicts that $z_{\text {int }}$ should be equal to that of the SW algorithm for the 3-state FM Potts model. Indeed, this is not surprising in the light of conjecture 1.1(b). 
Finally, it is worth mentioning that the convergence properties of the CP method are far better for critical points in the FM regime, than for those in the AF regime. Indeed, the estimates for the former critical points are several orders of magnitude more precise than the estimates for the latter. In addition, to our knowledge this is the first time that the location of the critical point for the 3-state FM Potts model on the $\mathcal{Q}$ (hextri) and $\mathcal{Q}$ (diced) have been computed to high precision.

\section{A Computation of the geometric factor}

The computation of the geometric factor $G$ for the $\mathcal{Q}$ (hextri) and $\mathcal{Q}$ (house) is not completely trivial (specially for the latter case).

Let us start with the simplest case: $\mathcal{Q}$ (hextri) shown in figure 15 . First we should find an appropriate way of representing this strip graph so that it is not distorted (like in figure $9(\mathrm{a})$ ). In this case the choice is obvious: take regular hexagons of lattice spacing $a$, so that all edges have the same length, except for the vertical edges splitting each hexagon into two equal halves (which have length $2 a$ ). The area covered by one application of the TM is given by $A_{L}=\frac{1}{2} d(1,3) d\left(1,1^{\prime}\right) L$, where the distances are $d(1,3)=2$, and $d\left(1,1^{\prime}\right)=2 a$ (see figure 15). Because $d(1,3)=\sqrt{3} a=2$, we get that $a=2 / \sqrt{3}$ and therefore, $A_{L}=(4 / \sqrt{3}) L$. This means that

$$
G(\mathcal{Q}(\text { hextri }))=\frac{4}{\sqrt{3}} .
$$

Notice that this is the right geometric factor for our definition of the TM and free energy density (3.23).

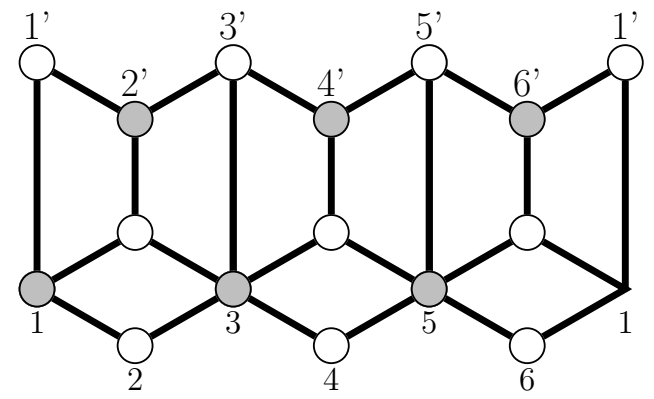

Figure 15: Strip of width $L=6$ of the lattice $\mathcal{Q}$ (hextri) with cylindrical boundary conditions and depicted in an non-distorted way (compare to figure 9(a)). An application of the TM transforms a bottom-row $(1,2, \ldots, L)$ configuration into a top-row $\left(1^{\prime}, 2^{\prime}, \ldots, L^{\prime}\right)$ configuration.

The second case $\mathcal{Q}$ (house) is more involved. Based on figure 2 of [59] (which represents the crystal structure of the $\mathrm{CrB}$ binary compound), we have produced figure 16. One of the house lattices is depicted with gray dots and thin solid blue 
lines, while its dual (which is also a house lattice) is displayed with white dots and thin dashed red lines. The quadrangulation $\mathcal{Q}$ (house) corresponds to both sets of dots and thick solid black lines. Notice that each house lattice contains triangular and pentagonal faces. In figure 2 of [59] the triangles are equilateral, and the pentagons are not regular: i.e., they are symmetric with respect to an axis going through the 'apex' vertex, and the two internal angles opposite to the apex vertex are equal to $\pi / 2$. The question is how to choose the free parameters in these lattices so we can get the physical value of $G$. Figure 16 shows a strip of width 6 and cylindrical boundary conditions, so that an application of the TM transforms the bottom-row $(1,2, \ldots, L)$ configuration into a top-row $\left(1^{\prime}, 2^{\prime}, \ldots, L^{\prime}\right)$ configuration. We have shown for clarity 'half' of the next row.

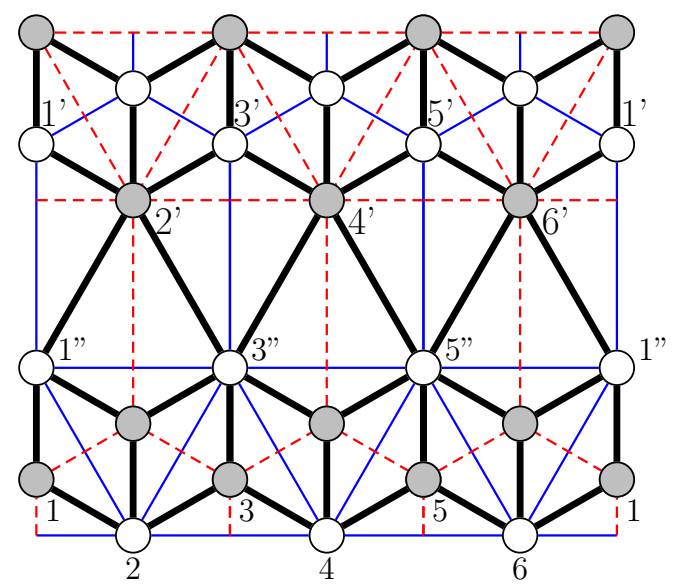

Figure 16: Strip of width $L=6$ of the lattice $\mathcal{Q}$ (house) with cylindrical boundary conditions and depicted in an non-distorted way (compare to figure 12). An application of the TM transforms a bottom-row $(1,2, \ldots, L)$ configuration into a top-row $\left(1^{\prime}, 2^{\prime}, \ldots, L^{\prime}\right)$ configuration. We also show the two underlying house lattices (white dots and thin solid blue lines, and gray dots and thin dashed red lines, respectively).

Again we should find an appropriate way of representing this strip graph so it is not distorted (like in figure 12). We can first assume that the triangles in both house lattices are equilateral with lattice spacing $d\left(1^{\prime \prime}, 3^{\prime \prime}\right)=d\left(3^{\prime \prime}, 2\right)=d\left(2,1^{\prime \prime}\right)=a$. The position of the dual vertex inside one triangular face should be placed at its barycenter. This fixes the position of the apex vertex of each pentagon (say, vertex 3 in figure 16). With this choice, the lattice spacing of the rhombi of the $\mathcal{Q}$ (house) lattice is given by $d\left(1,1^{\prime \prime}\right)=d(1,2)=a / \sqrt{3}$. We can see two rows formed by these rhombi at the bottom and at the top of the figure 16. However, the 'height' $b$ of the pentagon (i.e., the distance $\left.d\left(3^{\prime}, 3^{\prime \prime}\right)\right)$ remains unfixed. The only criterion we have found that makes sense is to choose $b$ so that the triangles formed by vertices $1^{\prime \prime}, 3^{\prime \prime}$ and $2^{\prime}$ (or, equivalently, $2^{\prime}, 4^{\prime}$ and $3^{\prime \prime}$ ) are also equilateral with lattice spacing equal to $a=d\left(1^{\prime \prime}, 3^{\prime \prime}\right)=d\left(2^{\prime}, 4^{\prime}\right)=d(2,4)$. This implies that $b=2 a / \sqrt{3}$. Note that with this choice, $\mathcal{Q}$ (house) is then nothing but an undistorted triangular lattice with extra 
vertices at the barycenters of every second row of triangles.

The area covered by one application of the TM is given by $A_{L}=\frac{1}{2} d(2,4) d\left(4,4^{\prime}\right) L$, where the distances are $d(2,4)=a=2$, and $d\left(4,4^{\prime}\right)=b+a / \sqrt{3}=a \sqrt{3}$ (see figure 16). Therefore, $A_{L}=(2 \sqrt{3}) L$. This means that

$$
G(\mathcal{Q}(\text { house }))=2 \sqrt{3} .
$$

Remark. In general, the determination of the geometrical factor $G$ amounts to determining which 'undistorted' representation of a given regular lattice will lead to a continuum limit having continuous rotational symmetry, which is clearly a minimum requirement for the critical model to acquire conformal invariance. However, the precise mechanism by which a 2, 3, 4 or 6-fold discrete rotational symmetry turns into a continuous one has not, to our knowledge, been much studied in the literature. In particular, the principle what constitutes an undistorted representation is not completely clear, except for the simplest Archimedean lattices.

The quadrangulation $\mathcal{Q}$ (house) considered above is a good case in point: The naive 2 -fold rotational symmetry is almost, but not quite, lifted to a 6 -fold symmetry by the choice (A.2). Indeed, in figure 16 every second horizontal row of triangles has an extra vertex inside each triangular face, while after a $\frac{\pi}{3}$ rotation, each horizontal row of triangles has an extra vertex inside every second triangular face. It is not clear how to obtain such a 'coarse grained' higher-order rotational symmetry for more general regular lattices.

\section{Acknowledgments}

This paper is dedicated to the memory of Chris Henley. We thank Alan Sokal for helpful comments and especially for sharing with us the initial ideas that led to this work and to reference [51]. We are also indebted to Kun Chen and Yuan Huang who were involved in the first stages of this work. Last but not least, we thank Gesualdo Delfino for correspondence and for pointing out to us reference [13].

JS is grateful for the hospitality of the Laboratoire de Physique Théorique at the École Normale Supérieure (from 2012 to 2018), and the Department of Mathematics at University College London (from 2012 to 2017), where part of this work was done.

This work has been supported in part by the National Natural Science Foundation of China under grants No. 11774002 (JPL), and No. 11625522 (YD), the Key Projects of Anhui Province University Outstanding Youth Talent Support Program grant gxyqZD2017009 (JPL), the Ministry of Science and Technology of China grant No. 2016YFA0301600 (YD), the Institut Universitaire de France, and the European Research Council through the Advanced Grant NuQFT (JLJ), and the MINECO FIS2014-57387-C3-3-P and MINECO/AEI/FEDER, UE FIS2017-84440-C2-2-P grants (JLJ and JS). 


\section{References}

[1] J. Adler, A. Brandt, W. Janke, and S. Shmulyian, J. Phys. A: Math. Gen. 28, $5117(1995)$.

[2] F. Alet, J.L. Jacobsen, G. Misguich, V. Pasquier, F. Mila, and M. Troyer, Phys. Rev. Lett. 94, 235702 (2005), arXiv: cond-mat/0501241.

[3] F. Alet, Y. Ikhlef, J.L. Jacobsen, G. Misguich and V. Pasquier, Phys. Rev. E 74, 041124 (2006), arXiv: cond-mat/0607747.

[4] J. Ashley, B. Grünbaum, G.C. Shephard and W. Stromquist, Self-duality groups and ranks of self-dualities, in Applied Geometry and Discrete Mathematics, DIMACS Series in Discrete Mathematics and Theoretical Computer Science, vol. 4 (American Mathematical Society, Providence RI, 1991), pp. 11-50.

[5] R.J. Baxter, Exactly Solved Models in Statistical Mechanics (Academic Press, London-New York, 1982).

[6] A. Bedini and J.L. Jacobsen, J. Phys. A: Math. Theor. 43, 385001 (2010), arXiv:1003.4847. Software package available for download at https://github. com/andreabedini/tutte.

[7] H.W.J. Blöte and B. Nienhuis, Phys. Rev. Lett. 72, 1372 (1984).

[8] H.W.J. Blöte and H.J. Hilhorst, J. Phys. A: Math. Gen. 15, L631 (1982).

[9] R. Burlisch and J. Stoer, Numer. Math. 6, 413 (1964).

[10] J.K. Burton Jr. and C.L. Henley, J. Phys. A: Math. Gen. 30, 8385 (1997), arXiv: cond-mat/9708171.

[11] M. Campostrini, A. Pelissetto, P. Rossi, and E. Vicari, Phys. Rev. E 57, 184 (1998), arXiv: cond-mat/9705086.

[12] Q.N. Chen, M.P. Qin, J. Chen, Z.C. Wei, H.H. Zhao, B. Normand, and T. Xiang, Phys. Rev. Lett. 107, 165701 (2011), arXiv:1105.5030.

[13] G. Delfino and E. Tartaglia, Phys. Rev. E 96, 042137 (2017), arXiv: 1707.00998.

[14] Y. Deng. Y. Huang, J.L.Jacobsen, J. Salas, and A.D. Sokal, Phys. Rev. Lett. 107, 150601 (2011), arXiv:1108.1743.

[15] P. Di Francesco, P. Mathieu and D. Sénéchal, Conformal Field Theory (SpringerVerlag, New York, 1997).

[16] S.J. Ferreira and A.D. Sokal, J. Stat. Phys. 96, 461 (1999), arXiv: cond-mat/9811345.

[17] C.M. Fortuin and P.W. Kasteleyn, Physica 57, 536 (1972). 
[18] T.M. Garoni, G. Ossola, M. Polin, and A.D. Sokal, J. Stat. Phys. 144, 459 (2011), arXiv:1105.0373.

[19] A. Ghosh, D. Dhar and J.L. Jacobsen, Phys. Rev. E 75, 011115 (2007), arXiv: cond-mat/0609322.

[20] B. Grünbaum and G.C. Shephard, Tilings and Patterns (W.H. Freeman and Co., New York, 1987).

[21] G. Haggard, D.J. Pearce, and G.F. Royle, ACM Transactions on Mathematical Software, 37, article 24 (2010). Software package available for download at http: //homepages.ecs.vuw.ac.nz/ djp/tutte/.

[22] C.L. Henley, Discrete Spin Models with 'Height' Representations and Critical Ground States, unpublished draft (September, 1993).

[23] C.L. Henley, J. Stat. Phys. 89, 483 (1997), arXiv: cond-mat/9607222.

[24] Y. Huang K. Chen, Y. Deng, J.L. Jacobsen, R. Kotecký, J. Salas, A.D. Sokal, and J. Swart, Phys. Rev. E 87, 012136 (2013), arXiv:1210.6248.

[25] D.A. Huse and A.D. Rutenberg, Phys. Rev. B 45, 7536 (1992).

[26] J.L. Jacobsen, J. Phys. A: Math. Theor. 40, 1439 (2007), arXiv:math-ph/0611078.

[27] J.L. Jacobsen, in Polygons, Polyominoes and Polycubes, edited by A.J. Guttmann, Lecture Notes in Physics \#775 (Springer, Dordrecht, 2009), Chapter 14 .

[28] J.L. Jacobsen, J. Phys. A: Math. Theor. 47, 135001 (2014), arXiv:1401.7847.

[29] J.L. Jacobsen, J. Phys. A: Math. Theor. 48, 454003 (2015), arXiv: 1507.03027.

[30] J.L. Jacobsen and J. Kondev, Nucl. Phys. B 532, 635 (1998), arXiv: cond-mat/9804048.

[31] J.L. Jacobsen and J. Salas, J. Stat. Phys. 104, 701 (2001), arXiv: cond-mat/0011456.

[32] J.L. Jacobsen, J. Salas and A.D. Sokal, J. Stat. Phys. 112, 921 (2003), arXiv: cond-mat/0204587.

[33] J.L. Jacobsen and J. Salas, Nucl. Phys. B 783, 238 (2007), arXiv: cond-mat/0703228.

[34] J.L. Jacobsen and J. Salas, unpublished (2008).

[35] J.L. Jacobsen and C.R. Scullard, J. Phys. A: Math. Theor. 45, 494003 (2012), arXiv: 1204.0622. 
[36] J.L. Jacobsen and C.R. Scullard, J. Phys. A: Math. Theor. 46, 075001 (2013), arXiv: 1211.4335.

[37] P.W. Kasteleyn and C.M. Fortuin, J. Phys. Soc. Japan 26 (Suppl.), 11 (1969).

[38] R. Kenyon, Ann. Prob. 28, 759 (2000).

[39] J. Kolafa, J. Phys. A: Math. Gen. 17, L777 (1984).

[40] J. Kondev, J. de Gier, and B. Nienhuis, J. Phys. A: Math. Gen. 29, 6489 (1994).

[41] J. Kondev and C.L. Henley, Phys. Rev. Lett. 73, 2786 (1994).

[42] J. Kondev and C.L. Henley, Phys. Rev. B 52, 6628 (1995).

[43] J. Kondev and C.L. Henley, Nucl. Phys. B 464, 540 (1996), arXiv: cond-mat/9511102.

[44] M. Korn and I. Pak, Theor. Comp. Sci. 319, 3 (2004).

[45] R. Kotecký, J. Salas and A.D. Sokal, Phys. Rev. Lett. 101, 030601 (2008), arXiv:0802.2270.

[46] R. Kotecký, A.D. Sokal and J.M. Swart, Commun. Math. Phys. 330, 1339 (2014), arXiv: 1205.4472.

[47] D.P. Landau and K. Binder, A Guide to Monte-Carlo Simulations in Statistical Physics, third edition (Cambridge Univesity Press, Cambridge, 2009).

[48] L.S. Levitov, Phys. Rev. Lett. 64, 92 (1990).

[49] E.H. Lieb, Phys. Rev. Lett. 18, 692 (1967).

[50] E.H. Lieb, Phys. Rev. 162, 162 (1967).

[51] J.-P. Lv, Y. Deng, J.L. Jacobsen, J. Salas, and A.D. Sokal, Phys. Rev. E 97, 040104(R) (2018), arXiv: 1712.07047.

[52] B. Mohar, Kempe equivalence of colorings, in Graph Theory in Paris, Proc. Conf. in Memory of Claude Berge, ed J.A. Bondy, J. Fonlupt, J.L. Fouquet, J.-C. Fournier and J. Ramírez Alfonsín (Birkhauser, Basel, 2006), pp. 287-297.

[53] B. Mohar and J. Salas, J. Phys. A: Math. Theor. 42, 225204 (2009), arXiv:0901.1010.

[54] B. Mohar and J. Salas, J. Stat. Mech.: Theor. Exper. P05016 (2010), arXiv: 1002.4279.

[55] J. Monroe, Phys. Rev. E 65, 066166 (2002).

[56] C. Moore and M.E.J. Newman, J. Stat. Phys. 99, 629 (2000), arXiv: cond-mat/9902295. 
[57] B. Nienhuis, J. Stat. Phys. 34, 731 (1984).

[58] M.P.M. den Nijs, M.P. Nightingale and M. Schick, Phys. Rev. B 26, 2490 (1982).

[59] M. O’Keeffe, Austral. J. Chem. 45, 1489 (1992).

[60] M. O'Keeffe and B.G. Hyde, Crystal Structures I. Patterns and Symmetry (Mineralogical Society of America, Washington DC, 1996), Section 5.3.7. Available on-line at http://www.public.asu.edu/ rosebudx/okeeffe.htm.

[61] H. Park and M. Widom, Phys. Rev. Lett. 63, 1193 (1989).

[62] R.B. Potts, Proc. Cambridge Philos. Soc. 48, 106 (1952).

[63] R. Raghavan, C.L. Henley, and S.L. Arouth, J. Stat. Phys. 86, 517 (1997), arXiv: cond-mat/9606220.

[64] J. Salas, J. Phys. A: Math. Gen. 31, 5969 (1998), arXiv: cond-mat/9802145.

[65] J. Salas and A.D. Sokal, J. Stat. Phys. 86, 551 (1997), arXiv: cond-mat/9603068.

[66] J. Salas and A.D. Sokal, J. Stat. Phys. 87, 1 (1997), arXiv:hep-lat/9605018.

[67] J. Salas and A.D. Sokal, J. Stat. Phys 92, 729 (1998), arXiv : cond-mat/9801079.

[68] J. Salas and A.D. Sokal, J. Stat. Phys. 104, 609 (2001), arXiv: cond-mat/0004330.

[69] C.R. Scullard, Phys. Rev. E 73, 016107 (2006), arXiv: cond-mat/0507392.

[70] C.R. Scullard and J.L. Jacobsen, J. Phys. A: Math. Theor. 45, 494004 (2012), arXiv:1209.1451.

[71] C.R. Scullard and J.L. Jacobsen, J. Phys. A: Math. Theor. 49, 125003 (2016), arXiv: 1511.04374.

[72] B. Servatius and H. Servatius, Symmetry, automorphisms, and self-duality of infinite planar graphs and tilings, in Proceedings of the International Scientific Conference on Mathematics (Žilina, 30 June - 3 July 1998), edited by V. Bálint (University of Žilina, Žilina, 1998), pp. 83-116. Available on-line at http:// users.wpi .edu/ bservat/self5.html.

[73] A.D. Sokal, in Functional Integration: Basics and Applications (1996 Cargèse summer school), C. DeWitt-Morette, P. Cartier, and A. Folacci, eds. (Plenum, New York, 1997).

[74] J. Stephenson, J. Math. Phys. 11, 1009 (1964).

[75] R.H. Swendsen and J.-S. Wang, Phys. Rev. Lett. 58, 86 (1987). 
[76] A. Sutö, Helv. Phys. Acta 84, 201 (1981).

[77] A. Sutö, Z. Phys. B 44, 121 (1981).

[78] W.P. Thurston, Amer. Math. Monthly 97, 757 (1990).

[79] J.-S. Wang, R.H. Swendsen and R. Kotecký, Phys. Rev. Lett. 63, 109 (1989).

[80] J.-S. Wang, R.H. Swendsen and R. Kotecký, Phys. Rev. B 42, 2465 (1990).

[81] J.C. Wierman, Construction of infinite self-dual graphs, in Proceedings of the 5th Hawaii International Conference on Statistics, Mathematics and Related Fields (2006).

[82] F.Y. Wu, Rev. Mod. Phys. 54, 235 (1982); 55, 315 (E) (1983).

[83] F.Y. Wu, J. Appl. Phys. 55, 2421 (1984).

[84] C. Zeng and C.L. Henley, Phys. Rev. B 55, 14935 (1997), arXiv: cond-mat/9609007.

[85] R.M. Ziff, C.R. Scullard, J.C. Wierman and M.R.A. Sedlock, J. Phys. A: Math. Theor. 45, 494005 (2012), arXiv:1210.6609. 